\title{
GALAXY ZOO: OBSERVING SECULAR EVOLUTION THROUGH BARS*
}

\author{
Edmond Cheung ${ }^{1}$, E. Athanassoula ${ }^{2}$, Karen L. Masters ${ }^{3,4,12}$, Robert C. Nichol ${ }^{3,4,12}$, A. Bosma ${ }^{2}$, Eric F. Bell ${ }^{5}$, \\ S. M. FAber ${ }^{1,6}$, David C. Koo ${ }^{1,6}$, Chris LintotT $^{7,8}$, Thomas Melvin $^{3}$, Kevin Schawinski $^{9}$, \\ Ramin A. SKIBbA ${ }^{10}$, AND Kyle W. Willett ${ }^{11}$ \\ ${ }^{1}$ Department of Astronomy and Astrophysics, 1156 High Street, University of California, Santa Cruz, CA 95064, USA; ec2250@ gmail.com \\ 2 Aix Marseille Université, CNRS, LAM (Laboratoire d'Astrophysique de Marseille) UMR 7326, F-13388, Marseille, France \\ ${ }^{3}$ Institute of Cosmology \& Gravitation, University of Portsmouth, Dennis Sciama Building, Portsmouth, PO1 3FX, UK \\ ${ }^{4}$ SEPnet, South East Physics Network, School of Physics and Astronomy, University of Southampton, Highfield, Southampton SO17 1BJ, UK \\ ${ }^{5}$ Department of Astronomy, University of Michigan, 500 Church Street, Ann Arbor, MI 48109, USA \\ ${ }^{6}$ UCO/Lick Observatory, Department of Astronomy and Astrophysics, University of California, 1156 High Street, Santa Cruz, CA 95064, USA \\ ${ }^{7}$ Oxford Astrophysics, Department of Physics, University of Oxford, Denys Wilkinson Building, Keble Road, Oxford OX1 3RH, UK \\ ${ }^{8}$ Astronomy Department, Adler Planetarium and Astronomy Museum, 1300 Lake Shore Drive, Chicago, IL 60605, USA \\ ${ }^{9}$ Institute for Astronomy, Department of Physics, ETH Zurich, Wolfgang-Pauli-Strasse 27, CH-8093 Zurich, Switzerland \\ ${ }^{10}$ Center for Astrophysics and Space Sciences, Department of Physics, 9500 Gilman Drive, University of California, San Diego, CA 92093, USA \\ ${ }^{11}$ School of Physics and Astronomy, University of Minnesota, MN 55455, USA \\ Received 2013 August 23; accepted 2013 October 10; published 2013 December 3
}

\begin{abstract}
In this paper, we use the Galaxy Zoo 2 data set to study the behavior of bars in disk galaxies as a function of specific star formation rate (SSFR) and bulge prominence. Our sample consists of 13,295 disk galaxies, with an overall (strong) bar fraction of $23.6 \% \pm 0.4 \%$, of which 1154 barred galaxies also have bar length (BL) measurements. These samples are the largest ever used to study the role of bars in galaxy evolution. We find that the likelihood of a galaxy hosting a bar is anticorrelated with SSFR, regardless of stellar mass or bulge prominence. We find that the trends of bar likelihood and BL with bulge prominence are bimodal with SSFR. We interpret these observations using state-of-the-art simulations of bar evolution that include live halos and the effects of gas and star formation. We suggest our observed trends of bar likelihood with SSFR are driven by the gas fraction of the disks, a factor demonstrated to significantly retard both bar formation and evolution in models. We interpret the bimodal relationship between bulge prominence and bar properties as being due to the complicated effects of classical bulges and central mass concentrations on bar evolution and also to the growth of disky pseudobulges by bar evolution. These results represent empirical evidence for secular evolution driven by bars in disk galaxies. This work suggests that bars are not stagnant structures within disk galaxies but are a critical evolutionary driver of their host galaxies in the local universe $(z<1)$.
\end{abstract}

Key words: galaxies: bulges - galaxies: evolution - galaxies: formation - galaxies: spiral - galaxies: statistics - galaxies: structure

Online-only material: color figures

\section{INTRODUCTION}

Stellar bar-shaped structures within galaxies, or more simply "bars," have been known to exist since the days of Edwin Hubble. With only the 100 inch $(2.54 \mathrm{~m})$ telescope at Mount Wilson, Hubble accurately surmised that bars were abundant in the local universe. Bars were so abundant that he devoted a major part of his classification scheme, the Hubble sequence of galaxies (Hubble 1936), to barred galaxies. Decades later, infrared and optical studies have confirmed that many galaxies have bars. Indeed, among local disk galaxies, as many as two-thirds are barred (e.g., Mulchaey \& Regan 1997; Knapen et al. 2000; Eskridge et al. 2000; Kormendy \& Kennicutt 2004; Menéndez-Delmestre et al. 2007; Sheth et al. 2008).

Bars have an important influence on galaxy evolution. The presence of bars has been linked to the existence of spiral arms, rings (Sanders \& Huntley 1976; Simkin et al. 1980; Schwarz 1981), and/or disky pseudobulges ${ }^{13}$ (Kormendy \& Kennicutt

\footnotetext{
* This publication has been made possible by the participation of more than 200,000 volunteers in the Galaxy Zoo project. Their contributions are individually acknowledged at http://www.galaxyzoo.org/Volunteers.aspx. 12 www.sepnet.ac.uk

13 Bulges created through secular evolution have been called both

"pseudobulges" and/or "disky bulges." For completeness, we will use the term "disky pseudobulges" throughout to represent such bulges in galaxies.
}

2004; Athanassoula 2005). Bars have also been associated with an increase in central star formation (Hawarden et al. 1986; Dressel 1988; Giuricin et al. 1994; Huang et al. 1996; Martinet \& Friedli 1997; Martin \& Friedli 1997; Ho et al. 1997; Ellison et al. 2011; Oh et al. 2012; Wang et al. 2012), the flattening of galactic chemical abundance gradients (Vila-Costas \& Edmunds 1992; Zaritsky et al. 1994; Martin \& Roy 1994; Williams et al. 2012), and, perhaps, active galactic nuclei (AGNs) activity (Noguchi 1988; Shlosman et al. 1989; Laine et al. 2002; Laurikainen et al. 2004; Martini et al. 2003; Jogee 2006; Hao et al. 2009; Oh et al. 2012).

Given that bars have an important influence on galaxy evolution, two natural questions are "How do bars form and evolve?" and "How do they affect their host galaxies?" A review of the theoretical work on bars is given by Athanassoula (2012), so we will only summarize here the parts that are most relevant to this work (see also Sellwood \& Wilkinson 1993; Sellwood 2013). Many past theoretical works have shown that bars can redistribute the angular momentum of the baryons and dark matter of a galaxy (e.g., Sellwood 1980; Debattista \& Sellwood 2000; Holley-Bockelmann et al. 2005). The angular momentum is emitted mainly by stars at (near) resonance in the bar region and absorbed mainly by (near) resonant material in the spheroid (i.e., the halo and, whenever relevant, the bulge) and in the outer disk (Lynden-Bell \& Kalnajs 1972; 
Tremaine \& Weinberg 1984; Athanassoula 2003, hereafter A03).

A03 showed that the redistribution of angular momentum is not merely a side effect of bars but is, instead, a process that is closely coupled to the evolution of bars. Specifically, the exchange of angular momentum from the inner disk to the outer disk and/or spheroid (bulge/halo) is the main driver of bar evolution. The efficiency of the angular momentum exchange is primarily dependent upon the mass distribution and velocity dispersion of the disk and spheroid. More angular momentum can be redistributed if the spheroid mass density at the location of the resonances is high, leading to stronger bars (A03). The second factor governing the efficiency of angular momentum exchange is the velocity dispersion. In lower velocity dispersion (lower temperature) disks and spheroids, resonances can emit or absorb more angular momentum than in cases with high velocity dispersion, thereby making the transfer of angular momentum more efficient (A03; Sheth et al. 2012).

This redistribution of angular momentum allows bars to drive gas and, to a lesser extent, stars to the centers of galaxies (Matsuda \& Nelson 1977; Simkin et al. 1980; Athanassoula 1992; Wada \& Habe 1992, 1995; Friedli \& Benz 1993; Heller \& Shlosman 1994; Knapen et al. 1995; Sakamoto et al. 1999; Sheth et al. 2005). This process is responsible for the increase of BL and strength and of the disk scale length (e.g., Hohl 1971; Debattista \& Sellwood 2000; A03; O’Neill \& Dubinski 2003; Valenzuela \& Klypin 2003; Debattista et al. 2006; MartinezValpuesta et al. 2006; Minchev et al. 2011), the formation of a disky pseudobulge (Kormendy \& Kennicutt 2004; Athanassoula 2005), the increase of central star formation (Friedli \& Benz 1993; Martinet \& Friedli 1997; Martin \& Friedli 1997), and the dilution of abundance gradients (Friedli et al. 1994; Friedli \& Benz 1995; Martel et al. 2013). This process is known as secular evolution (Kormendy \& Kennicutt 2004; Kormendy 2012).

It has been shown that bar formation and evolution is also dependent on the gas content in the galaxy (e.g., Shlosman \& Noguchi 1993; Berentzen et al. 1998, 2007; Villa-Vargas et al. 2010). More recent simulations - with a multiphase description of the gas, including star formation, feedback and cooling, and a sufficiently large number of particles to describe adequately the gas flow-have shown that bars form later in simulations with a larger gas fraction (Athanassoula et al. 2013, hereafter AMR13).

Recent observational works have begun to test many of these predictions. For example, Masters et al. (2011) used classifications from GZ2 (see Section 2.2) to show that the fraction of disk galaxies that possess a bar (bar fraction) increases in redder disk galaxies (see also Skibba et al. 2012). This result was confirmed by Lee et al. (2012), who also used a large sample of galaxies from the Sloan Digital Sky Survey (SDSS), but with their own classifications (combining a mix of visual and automated methods). Assuming that galaxy color is closely related to galactic gas content (e.g., Catinella et al. 2010; Saintonge et al. 2011), then this is consistent with the expected effects of gas on bar formation and evolution. Indeed, using a sample of GZ2 bars with $\mathrm{H}$ I measurements from the ALFALFA survey, Masters et al. (2012) found that the bar fraction correlates strongly with H I content. In that sample, more bars were found in the gas-poor disk galaxies, even at fixed color or stellar mass.

Alternatively, Barazza et al. (2008) and Aguerri et al. (2009) found different results using samples of SDSS galaxies with bars identified from ellipse fitting methods. Both of these works found that bar fractions were larger for the bluer (and presumably more gas rich) galaxies in their samples. However, Nair \& Abraham (2010a, 2010b) suggest a way to reconcile these results that came from samples of disk galaxies with very different selections; notably, Barazza et al. 2008 and Aguerri et al. 2009 selected only blue galaxies as disks and included lower redshift and less massive galaxies than were present in Masters et al. (2011), Masters et al. (2012), or Lee et al. (2012). The sample of Nair \& Abraham (2010a), which probed a wide range of stellar mass, suggested that bar fraction is bimodal with disk galaxy color, having peaks both toward the bluer and redder disk galaxies. ${ }^{14}$ Nair \& Abraham (2010a) suggest this trend may reveal two distinct types of bars; namely, weak bars are predominantly found in lower-mass and more gas-rich (and bluer) spirals, while stronger bars are more common in massive, redder, and gas-poor disks.

In addition to the dependence on galaxy color, bar fraction has also been found to depend on inner galactic structure. Masters et al. (2011) found that bar fraction was correlated with fracDeV, which is a parameter measured by the SDSS representing the fraction of the best-fit light profile that originates from the de Vaucouleurs fit to the profile, as opposed to an exponential fit. Lee et al. (2012) also found that the bar fraction was highest at moderate central velocity dispersion. However, Barazza et al. (2008) found that barred galaxies are most likely to exist in galaxies with low Sérsic indices, while Aguerri et al. (2009) found that bars are most likely to exist in galaxies with low concentration indices. Although these results appear to be conflicting, they all show that the presence of a galactic bar is influencing the inner structure of these galaxies.

While the trends of bar fraction can reveal aspects of bar formation and evolution, bar fraction is crude as it hides information on the bar itself. According to A03, the characteristics of a bar (e.g., long or short) can be used as tracers of bar evolution. Therefore, a common bar property that has been studied in the literature is BL. Athanassoula \& Misiriotis (2002) and A03 predicted that the presence of a bulge will result in a longer and more evolved bar. Comparing this prediction to previous observational works shows a good consensus; early-type disk galaxies do indeed have longer bars (Kormendy 1979; Athanassoula \& Martinet 1980; Martin 1995; Elmegreen \& Elmegreen 1985; Regan \& Elmegreen 1997). Larger samples and/or infrared imaging continues this agreement (Laurikainen et al. 2002, 2007; Erwin 2005; Menéndez-Delmestre et al. 2007; Elmegreen et al. 2007; Aguerri et al. 2009; Gadotti 2011; Hoyle et al. 2011).

In this paper, we use the GZ2 data set (Masters et al. 2011; Hoyle et al. 2011; Willett et al. 2013) to investigate how the likelihood of a galaxy hosting a galactic bar depends on two important factors, namely, the gas content of the galaxy and its inner galactic structure. We perform the same investigation with $\mathrm{BL}$ and compare both of these sets of relationships to theoretical predictions, which will give us not only a better understanding of bar formation and evolution but also a better understanding of how bars affect their host galaxy.

We begin in Section 2 by describing all the data used in the paper, while the main observational results are presented in Section 3. We compare our results with several theoretical simulations in Section 4 and discuss our work and these comparisons in Section 5. We conclude in Section 6. In Appendix A, we discuss the completeness of our sample. We assume a cosmological model with $H_{0}=70 \mathrm{~km} \mathrm{~s}^{-1} \mathrm{Mpc}^{-1}$, $\Omega_{m}=0.30$, and $\Omega_{\Lambda}=0.70$ throughout this paper.

\footnotetext{
14 Masters et al. (2011) also commented on a possible upturn in bar fraction for the bluest galaxies in their sample.
} 
Table 1

Sample Selection

\begin{tabular}{lcc}
\hline \hline Criterion & GZ2D & BL \\
& No. & No. \\
\hline Galaxy Zoo 2 & 295,305 & 3150 \\
$0.01<z<0.06^{\mathrm{b}}$ & 76,336 & 2674 \\
$M_{r}<-20.15$ & 43,266 & 2177 \\
$b / a>0.5$ & 28,540 & 1753 \\
$1 / 4$ answers bar question & 14,353 & 1753 \\
$p_{m g}<0.4$ & 14,038 & 1734 \\
GIM2D models $<1^{\prime \prime}$ offset & 13,328 & 1655 \\
Quality GIM2D disks & 13,328 & 1159 \\
MPA cross-match & 13,295 & 1154 \\
\hline
\end{tabular}

Notes.

${ }^{a}$ See footnote 18 .

b We only consider galaxies with spectroscopic redshifts.

\section{DATA}

This section lists all sources of data that this paper uses. In order to have a fully complementary data set, we cross-matched every data set, as described in each subsection, resulting in a successive reduction of the initial sample size. As a guide, our initial data set is described in Section 2.2, which derives from SDSS Data Release Seven (DR7) (summarized in Section 2.1). We list the sample totals at the end of each subsection, starting with Section 2.2. Table 1 lists every major cut made to our two samples and the resultant sample sizes.

\subsection{SDSS}

All the galaxies used in our sample are drawn from the main galaxy sample in the legacy area of the SDSS DR7 (Strauss et al. 2002; Abazajian et al. 2009). Where possible, we use the standard photometric and structural parameters provided by the SDSS pipeline. For example, we use the SDSS information to define a surface stellar mass density within a radius of $1 \mathrm{kpc}$ of the center of the galaxy, $\Sigma_{1 \mathrm{kpc}}^{*}$. We choose $1 \mathrm{kpc}$ for this density as it matches the typical scale of bulges (Fisher \& Drory 2010) and therefore should be closely related to the bulges of most galaxies.

In detail, $\Sigma_{1 \mathrm{kpc}}^{*}$ is created from the SDSS galaxy surface brightness profiles, profMean, which is the mean surface brightness in a series of circular annuli, from the PhotoProfile table in the CasJobs Web site. ${ }^{15}$ In accordance with the SDSS recommendations, ${ }^{16}$ we take the inverse hyperbolic sine of each cumulative profile and fit it with a natural cubic spline. After transforming the spline fits back with a sine function, we differentiate the fits and obtain an estimate of the azimuthally averaged surface brightness profile. Finally, we compute the magnitude and color within $1 \mathrm{kpc}$ for each galaxy from these profiles and convert them into a stellar mass through a colordependent mass-to-light $\left(M_{*} / L_{g}\right)$ ratio (e.g., Bell \& de Jong 2001). Our $M_{*} / L_{g}$ relationship is derived from a linear fit to the rest-frame $g-r$ color from GIM2D (see Section 2.3) and $M_{*} / L_{g}$, where the stellar masses are taken from the MPA-JHU catalog (see Section 2.4) and the $g$-band luminosity is taken from GIM2D models.

\footnotetext{
15 http://casjobs.sdss.org/CasJobs/

16 http://www.sdss.org/dr7/algorithms/photometry.html
}

One concern is that the $1 \mathrm{kpc}$ radius aperture is smaller than the typical seeing of SDSS. However, an analysis of angular sizes of galaxies in our sample, which lies within the redshift range $0.01<z<0.06$ (see Section 2.2), shows they are typically larger than the full width at half maximum of the SDSS point-spread function $\left(\sim 1^{\prime \prime} .3\right.$ in the $r$ band; Abazajian et al. 2009).

\subsection{Galaxy Zoo}

Galaxy Zoo (GZ) is a citizen science project that enlisted hundreds of thousands of volunteer "citizen scientists" to make morphological classifications of nearly a million galaxies (Lintott et al. 2008, 2011). The initial GZ project asked the public to classify galaxies as elliptical, spiral, or merger. With the GZ2 project (Willett et al. 2013), the citizen scientists were asked to make more detailed classifications of approximately 304,000 galaxies.

The final product of GZ2 is a table of morphological likelihoods, including the likelihood that a bar is present in each galaxy, as represented by $p_{\text {bar }}$, e.g., if 5 out of 10 scientists classified a galaxy as having a bar, the galaxy would be assigned a bar probability of $p_{\text {bar }}=0.5$. These raw probabilities are then adjusted to account for the reliability of each user through an iterative weighting scheme that "down-weights" classifications from unreliable users (typically, a few percent of the population). We also apply a correction to the likelihoods to account for the deterioration of the image quality due to increasing distance of galaxies; that is, we assume galaxies of a similar luminosity and size will share the same average mix of morphologies regardless of redshifts. This also assumes there is no significant evolution within the SDSS at these low redshifts, which is probably reasonable (Bamford et al. 2009; Willett et al. 2013). Therefore, throughout this paper, we will only use these corrected, or "debiased," bar likelihoods and will calling them $p_{\text {bar }}$ for convenience.

In Masters et al. (2011) and Masters et al. (2012), barred galaxies were selected using $p_{\text {bar }} \geqslant 0.5$. This threshold delivered a high purity of barred galaxies in comparison with other barred galaxy samples; for example, almost all galaxies with $p_{\text {bar }} \geqslant 0.5$ were classified as possessing a strong bar by Nair \& Abraham (2010a, see Appendix A of Masters et al. 2012). Weaker bars in Nair \& Abraham (2010a) were found to correspond to $0.3 \leqslant p_{\text {bar }} \leqslant 0.5$ (Masters et al. 2012; Willett et al. 2013).

In this work, we choose to use $p_{\text {bar }}$ as a bar likelihood, rather than as a bar threshold. This method has been used before with GZ classifications (e.g., Bamford et al. 2009; Skibba et al. 2009, 2012). Our results are in qualitative agreement with other GZ results that used bar fractions; for example, if we adopt a bar threshold of $p_{\text {bar }}=0.5$, we find an overall bar fraction of $23.6 \% \pm 0.4 \%$, which is similar to Masters et al. (2011). ${ }^{17}$

Our initial sample is the GZ2 data set. ${ }^{18}$ Following Masters et al. (2011), we only select galaxies with spectroscopic redshifts in the range of $0.01<z<0.06$. In order to have a volumelimited sample, we only include galaxies with $M_{r}<-20.15$, where $M_{r}$ is the rest-frame absolute Petrosian $r$-band magnitude. This limit corresponds to the GZ2 completeness Petrosian

\footnotetext{
17 The difference between our bar fraction and that of Masters et al. (2011) is due to the use of the weighted and debiased bar fractions from Willett et al. (2013), which were unavailable at the time of Masters et al. (2011).

18 This Galaxy Zoo 2 sample is comprised of the "original," "extra," and "stripe82" samples in Table of Willett et al. (2013). These data are available at http://data.galaxyzoo.org.
} 
magnitude of 17 in the $r$ band (Willett et al. 2013) at $z=0.06$. To ensure that our sample contains relatively face-on galaxies, we applied an axis ratio requirement of $b / a>0.5$ (this corresponds to inclination angles less than $\approx 60^{\circ}$ ), where $b / a$ is the axis ratio from the GIM2D single Sérsic model fit (see Section 2.3). This requirement minimizes projection effects and thus results in more reliable bar classifications. This sample also requires that all galaxies have a Petro90 radius of $>3^{\prime \prime}$. We have tested our results with a larger minimum radius requirement and find that our results are unchanged. ${ }^{19}$

We also require that for each galaxy, at least a quarter of all its classifications involved answering the bar question, "Is there a sign of a bar feature?" (Masters et al. 2011). In order to reach the bar question, however, a user must first classify a galaxy as a non-edge-on galaxy with a disk or some sort of feature (e.g., spiral arms, rings, bars). Assuming that most identified features are associated with a disk, then this last selection effectively ensures we have non-edge-on disk galaxies.

Finally, we discard all merging galaxies from the sample since we are only concerned with isolated galaxies that have reliable photometric and structural measurements. According to Darg et al. (2010), the GZ merging parameter $p_{\mathrm{mg}}$ can identify merging galaxies with a cut of $p_{\mathrm{mg}}>0.4$; we adopt this threshold to eliminate merging galaxies. There is a total of 14,038 galaxies in the resulting sample, which we will call the Galaxy Zoo 2 Disk (GZ2D) sample.

We carefully review here the makeup of our sample to avoid confusion with comparisons with other disk, spiral, or latetype selections based on GZ morphologies. The disk galaxy selection presented herein possibly includes a fraction of very early-type disks galaxies (Sa or S0), which would normally be included in a majority of early-type samples selected either by color or central concentration. This results in our diverse disk galaxy sample showing bimodality in its optical color-mass diagram (Figure 3(a)). However, other GZ samples that are more focused on late-type disks or spirals sample ( $\mathrm{Sb}, \mathrm{Sc}$, or later) can be constructed using the GZ1 "clean" spiral criterion as first discussed in Land et al. (2008) and most recently used in Schawinski et al. (2013) and also through stricter limits in GZ2/ GZ Hubble data. This more conservative late-type sample will be more dominated by "blue cloud" spirals and thus will show less bimodality of its galaxy properties.

In addition to this sample, we use a GZ2 subsample that possesses additional BL measurements. The BLs were visually measured by citizen scientists using a Google Maps interface described by Hoyle et al. (2011). The bar lengths represent the lengths from one end of the bars to the other. In order to be consistent with previous works, which define it as the semimajor axis of maximum ellipticity in the bar region (e.g., Erwin 2005), we will take half of the GZ2 bar length and denote it $L_{\text {bar }}$. This catalog requires at least three independent BL measurements per galaxy; the mean of these independent BL measurements gives $L_{\text {bar }}$ of each galaxy. The vast majority of galaxies that were selected for this sample have $p_{\text {bar }} \geqslant 0.6$; that is, this sample contains mainly strong bars (Masters et al. 2012; Willett et al. 2013). Of the GZ2D sample, there are 1734 galaxies that have BL measurements, which will now be referred to as the BL sample.

We present a gallery of barred galaxies with a range of $p_{\text {bar }}$ and $L_{\text {bar }}$ in Figure 1. Each row is ordered by absolute BL.

\footnotetext{
19 We find that our results are unchanged when we restrict our sample to galaxies with global half-light radii (as measured by GIM2D) larger than $5^{\prime \prime}$.
}

\subsection{GIM2D}

Two-dimensional bulge+disk decompositions in the $g$ and $r$ bandpasses of over a million SDSS galaxies were performed with GIM2D by Simard et al. (2011). Improvements to the sky background determinations and object deblending over the standard SDSS procedures led to more robust galactic structural parameters than those offered by the standard SDSS pipeline.

Three different models were used in these decompositions: a pure Sérsic model, an $n=4$ bulge+exponential disk model, and a Sérsic (free-floating $n$ ) bulge+exponential disk model. The most important GIM2D parameter for the GZ2D sample is the galaxy Sérsic index $n$ from the pure Sérsic model, i.e., the bestfitting single Sérsic index for a given galaxy. The Sérsic index has often been used to separate early-type and late-type galaxies and is widely regarded as a good proxy for bulge dominance (Blanton et al. 2003; Shen et al. 2003; Bell et al. 2004; Bell et al. 2012; Schiminovich et al. 2007; Drory \& Fisher 2007; Bell 2008; Wuyts et al. 2011; Wake et al. 2012; Cheung et al. 2012).

Although a similar parameter, fracDeV from the SDSS database, has been explored by previous works (e.g., Masters et al. 2011; Skibba et al. 2012), $n$ is a more common parameter in the literature and has been thoroughly studied (e.g., Graham \& Driver 2005). It is also the basis of most galaxy fitting programs (e.g., GALFIT, BUDDA, and GIM2D; Peng et al. 2002; de Souza et al. 2004; Simard et al. 2002), which allows for easier and more consistent comparisons to other works. For reference, we compare $n$ and fracDeV in Figure 2 for our GZ2D sample. Clearly, the two parameters are correlated. However, the overdensity of galaxies at fracDeV $=1$, which accounts for $\sim 15 \%$ of the GZ2D sample, indicates that there is a saturation of galaxy structural information in the fracDeV parameter. Indeed, for fracDeV $=1, \log n$ ranges from 0.5 to 0.9 , corresponding to $n \sim 3-8$. A similar effect occurs at fracDeV $=0$, which accounts for another $\sim 12 \%$ of the GZ2D sample. Our use of the Sérsic index in this paper should be more sensitive than fracDeV to the complicated structures of galaxies.

Another similar parameter is the Petrosian concentration index from SDSS. This parameter has been shown by Gadotti (2009) to be a better proxy for bulge fraction than the global Sérsic index. We would like to note, however, that the global Sérsic indices that Gadotti (2009) used were from the New York University (NYU) Value-Added Galaxy Catalog (Blanton et al. 2005a, 2005b), which fitted one-dimensional profiles extracted from two-dimensional images using circular annuli. The GIM2D fits were done using elliptical annuli and are two-dimensional fits. As noted by Simard et al. (2011), this difference in methodology, i.e., using circular and elliptical apertures, results in a systematic offset between the NYU and GIM2D galaxy half-light radius and galaxy Sérsic index. At the request of the referee, we tested our results using R90/R50 in Appendix $\mathrm{C}$-we find no major impacts to our conclusions.

The most important GIM2D parameter for the BL sample is the semimajor axis exponential disk scale length $r_{\mathrm{d}}$; this is needed to properly scale the BL. The disk scale length is available in both the $n=4$ bulge+exponential disk model and the Sérsic bulge+exponential disk model; we use the latter model. ${ }^{20}$ As is noted in Simard et al. (2011), the quality of the GIM2D bulge+disk decompositions is highly dependent on the spatial resolution and signal-to-noise ratio of the SDSS images.

\footnotetext{
20 We find no change in our main conclusions if we use the $n=4$
} bulge+exponential disk model. 


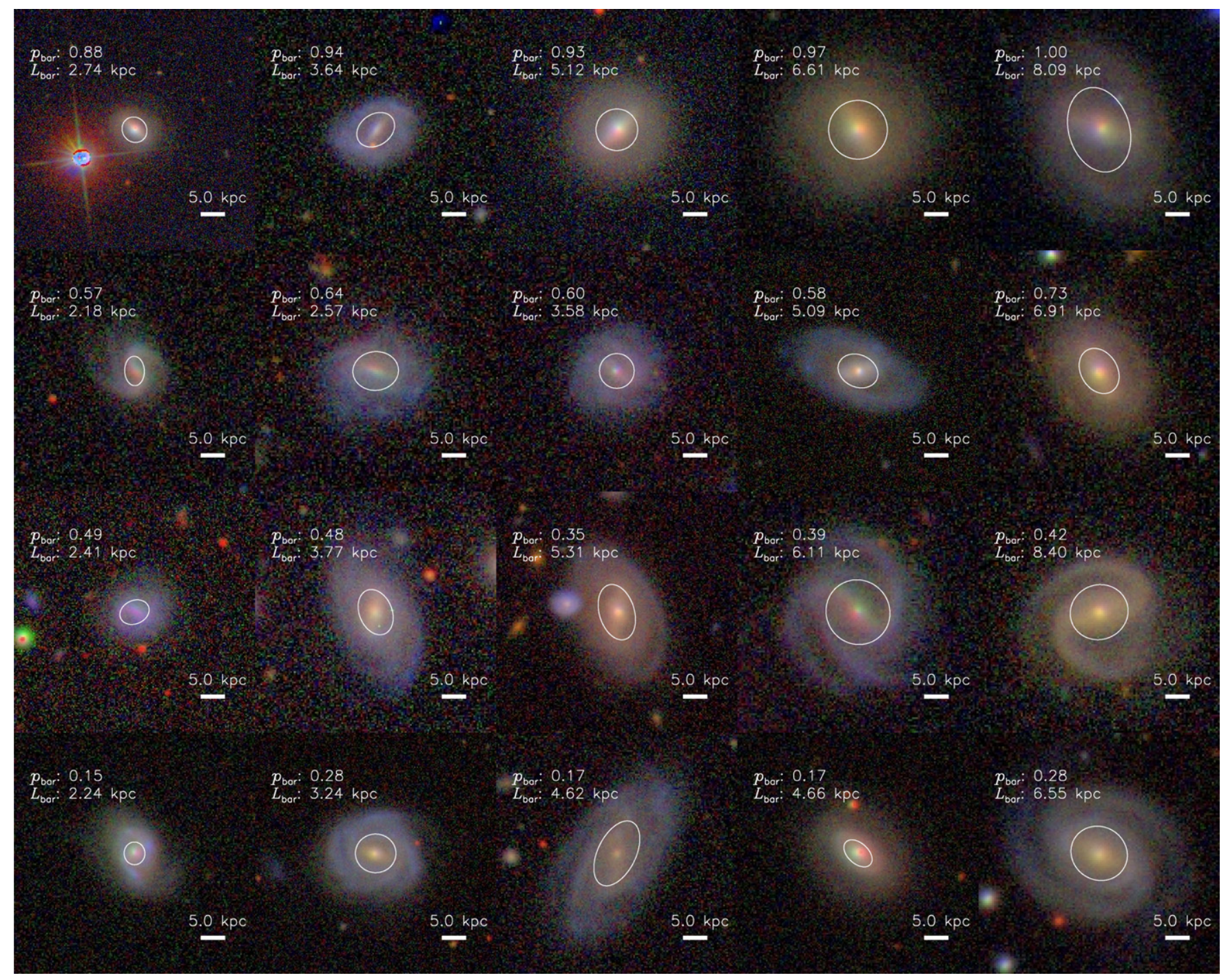

Figure 1. Gallery of galaxies with a range of bar likelihood $p_{\mathrm{bar}}$ and bar length $L_{\mathrm{bar}}$. Each row is ordered by increasing bar length. The ellipse drawn over each galaxy represents the GIM2D disk model at $r_{\mathrm{d}}$. Visually, the bars generally extend out the disk scale length, consistent with Combes \& Elmegreen (1993). The physical scales of every image are the same ( \pm 1 pixel)

(A color version of this figure is available in the online journal.)

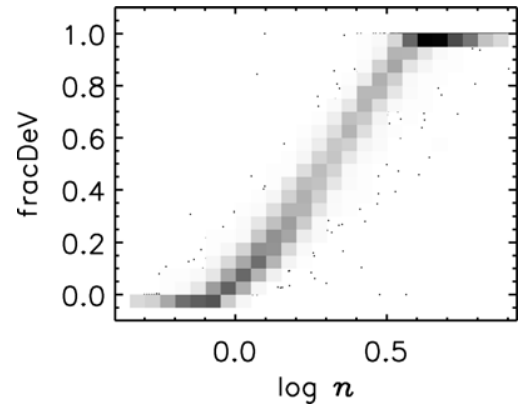

Figure 2. Comparison of $n$ from the GIM2D single Sérsic model fit and fracDeV ( $r$ band) from the SDSS database. The cluster of galaxies at fracDeV $=1$ and fracDeV $=0$ accounts for $\sim 27 \%$ of the total GZ2D sample, indicating that there is a loss of galaxy structural information in the fracDeV parameter.

Therefore, it is important to ensure that we only allow model fits that are reliable. However, since we are only concerned with $r_{\mathrm{d}}$, picking out reliable decompositions is not difficult. From L. Simard (2012, private communication), galaxy models with $B / T \leqslant 0.5$ (the $B / T$ from the Sérsic bulge+exponential disk model) accurately model the disk component, and therefore we consider all these galaxies. This is understandable since these galaxies are disk dominated, and their corresponding GIM2D models will likely yield reliable disk measurements. For models with $B / T>0.5$, L. Simard (private communication) recommends considering only galaxies with $P_{\mathrm{pS}}<0.32$, where $P_{\mathrm{pS}}$ represents the probability that a bulge+disk model is $n o t$ required compared to a pure Sérsic model (Simard et al. 2011). Thus $B / T>0.5$ galaxies that have a high probability of requiring a bulge+disk model are also considered.

To avoid the effects of the SDSS point-spread function on the GIM2D disk model, we only allow disk models with $r_{\mathrm{d}}>2^{\prime \prime}$. Furthermore, we impose a strict face-on requirement such that all GIM2D model disks have inclination angles of less than $55^{\circ}$. This corresponds to axis ratios greater than 0.6 , a parameter space that has been shown by MacArthur et al. (2003) to produce no systematic variations on $r_{\mathrm{d}}$ when using two-dimensional galaxy decompositions. Finally, we require that the fractional errors on $r_{\mathrm{d}}\left(r_{\mathrm{d} \text {,error }} / r_{\mathrm{d}}\right.$, where $r_{\mathrm{d} \text {,error }}$ is the formal error of $r_{\mathrm{d}}$ from GIM2D) be less than $2 \%$. This number is approximately two standard deviations above the average $r_{\mathrm{d}, \text { error }} / r_{\mathrm{d}}$ of the $\mathrm{BL}$ 


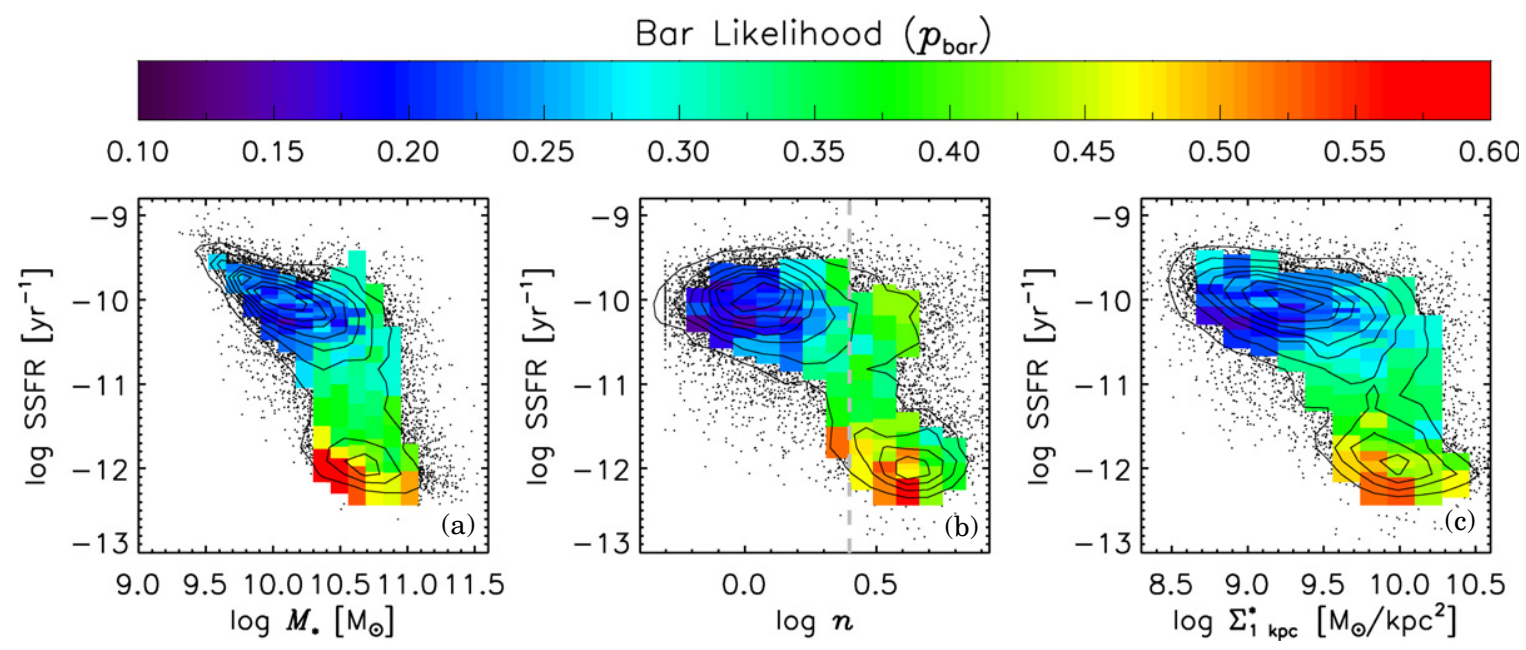

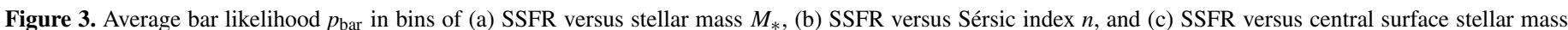

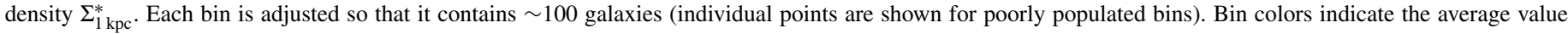

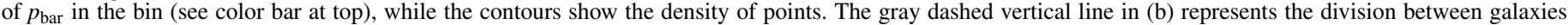

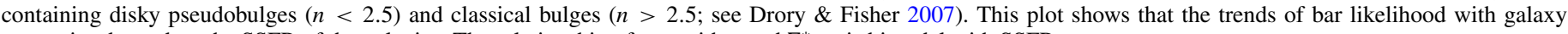
properties depend on the SSFR of the galaxies. The relationship of $p_{\text {bar }}$ with $n$ and $\Sigma_{1 \mathrm{kpc}}^{*}$ is bimodal with SSFR.

(A color version of this figure is available in the online journal.)

sample. We choose this conservative cut in order to include only quality disk models.

We note that although we only model a bulge and disk for these disk galaxies, $\sim 24 \%$ of which are strongly barred, previous works have shown that while bulge parameters may be affected by the presence of a bar, the disk scale length is not significantly affected (Erwin 2005; Laurikainen et al. 2005). This reliability is evident in the fact that our results are not sensitive to the choice of GIM2D bulge+disk model; that is, $r_{\mathrm{d}}$ from both the $n=4$ bulge+disk model and the Sérsic bulge+disk model produce the same results. Furthermore, the GIM2D formal errors on $r_{\mathrm{d}}$ are not significantly different from strongly barred systems $\left(p_{\text {bar }}>0.8\right)$ and nonbarred systems $\left(p_{\text {bar }}<0.05\right)$.

We impose a final cut that eliminates all GIM2D models where the centers are offset from the input science images by more than $1^{\prime \prime}$. Large offsets like these usually represent a bad fit, and, indeed, upon visual inspection, we find that almost all these cases contained bright point sources within the galaxy and/or diffraction spikes from nearby stars. Matching the GZ2D and BL samples to the GIM2D catalogs leaves us with 13,328 and 1,159 disk galaxies, respectively.

\subsection{MPA-JHU}

Stellar masses and star formation rates (SFRs) are taken from the MPA-JHU DR7 release. ${ }^{21}$ Stellar mass $\left(M_{*}\right)$ estimates are calculated using the Bayesian methodology and model grids described in Kauffmann et al. (2003). The models are fit to the broadband ugriz SDSS photometry, instead of the spectral indices from the $3^{\prime \prime}$ fiber aperture. These estimates are corrected for nebular emission, and a Kroupa (2001) initial mass function is assumed.

SFR are based on the technique presented in Brinchmann et al. (2004). For their "star-forming" class, which consists of 39,141 galaxies, they estimate the SFR from model fits

\footnotetext{
21 http://www.mpa-garching.mpg.de/SDSS/DR7/
}

that cover a wide range of star formation histories of several emission lines from the SDSS fiber. For the "low S/N starforming" class, which contains 29,115 galaxies, they convert the observed $\mathrm{H} \alpha$ luminosity into an SFR. And for the "AGN," "composite," and "unclassifiable" classes, which contain a total of 66,986 galaxies, they use the D4000 value to estimate SFR/ $M_{*}$ and SFR. Aperture corrections follow the method of Salim et al. (2007), resulting in the SFR of the entire galaxy. The specific star formation rate (SSFR), a parameter that will be used throughout this paper, is defined as the SFR divided by stellar mass; it was calculated by combining the SFR and $M_{*}$ likelihood distributions as outlined in Appendix A of Brinchmann et al. (2004).

Matching the GZ2D and BL samples to the MPA-JHU catalog brings our final sample to 13,295 and 1,154 , respectively. A detailed discussion of the completeness of the GZ2D and BL samples is presented in Appendix A. We find that although we are missing some low-mass quiescent disk galaxies, the effect is small and does not affect our results.

\section{RESULTS}

\subsection{Bar Likelihood Trends}

In this section, we present the trends of bar likelihood with SSFR, stellar mass, and measures of bulge prominence.

The three panels of Figure 3 plot both galaxy density and average $p_{\text {bar }}$ in a two-dimensional plane of SSFR versus stellar mass $\left(M_{*}\right.$; panel (a)), SSFR versus global Sérsic index $(n$; panel (b)), and SSFR versus central surface stellar mass density $\left(\Sigma_{1 \mathrm{kpc}}^{*}\right.$; panel (c)). The locations of the galaxies are shown by the contours. Bin sizes are adjusted so that they contain $\sim 100$ galaxies each, and individual data points are shown for poorly populated bins. Each bin is colored by the average $p_{\text {bar }}$ of the galaxies in it, as indicated by the color bar.

The well-known bimodality between galaxies (even for disk galaxies) in the star-forming sequence and those in the quiescent population is clear in our sample and affects not only the 

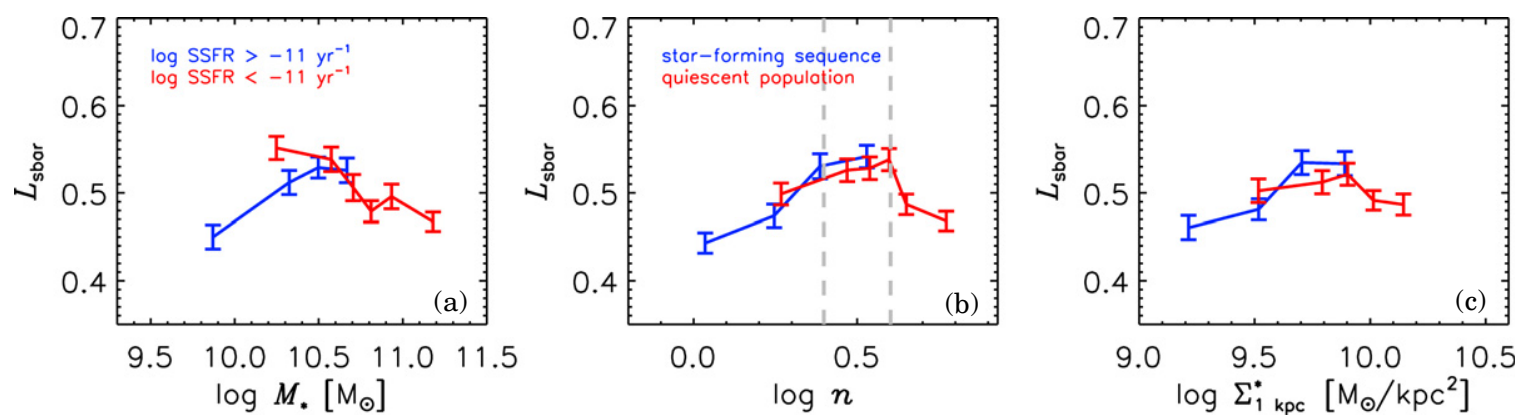

Figure 4. Average $L_{\mathrm{sbar}}$ plotted against (a) $M_{*}$, (b) $n$, and (c) $\Sigma_{1 \mathrm{kpc}}^{*}$. Galaxies were split by their star formation state, namely, log SSFR $>-11 \mathrm{yr}^{-1}$ (star forming; blue) and $\log$ SSFR $<-11 \mathrm{yr}^{-1}$ (quiescent; red). Each bin contains $\sim 100$ galaxies. The error bars are given by $\sigma / \sqrt{N}$, where $\sigma$ is the standard deviation of $L_{\mathrm{sbar}}$ per bin and $N$ is the total number of galaxies per bin. The vertical dashed lines in (b) are located at $\log n=0.4(n=2.5)$ and $\log n=0.6(n=4)$.

(A color version of this figure is available in the online journal.)

galaxies' SSFR, baryonic mass, and bulge properties but also their likelihood of being barred (Masters et al. 2011). We find that there is a strong correlation between average $p_{\text {bar }}$ and SSFR such that the average values of $p_{\text {bar }}$ are larger for low-SSFR disk galaxies (i.e., quiescent disk galaxies are more likely to host bars). The observed relationship between $p_{\text {bar }}$ and SSFR is present even at fixed $M_{*}, n$, or $\Sigma_{1 \mathrm{kpc}}^{*}$ (Figure 3 ), indicating that this relationship is nearly independent of these galaxy properties.

Taking SSFR as a proxy for gas fraction (e.g., Kauffmann et al. 2012) suggests that the underlying relationship is really between $p_{\text {bar }}$ and gas content such that bar likelihood is increasing as gas fraction decreases. Similar trends between bar fraction and gas content were also observed by Masters et al. (2012).

We observe that the trends of the average bar likelihood with $M_{*}, n$, and $\Sigma_{1 \mathrm{kpc}}^{*}$ depend on whether the disk galaxy is star forming or quiescent, as illustrated by Figure 3. Thus, we look in more detail at the observed trends within the star-forming $\left(\log\right.$ SSFR $\left.>-11 \mathrm{yr}^{-1}\right)$ and quiescent $\left(\log\right.$ SSFR $\left.<-11 \mathrm{yr}^{-1}\right)$ disk galaxy populations. We find the following.

1. Stellar mass $M_{*}$ (Figure 3(a)). There is a correlation between average $p_{\text {bar }}$ and stellar mass within the starforming disks such that $p_{\text {bar }}$ is larger the larger their stellar mass is. There is also an anticorrelation of $p_{\text {bar }}$ with stellar mass within the quiescent population.

2. Sérsic index n (Figure 3(b)). For the star-forming sequence, $p_{\text {bar }}$ is strongly correlated with $n$ (even more so than it is with $M_{*}$ ). Within the quiescent population, we see an inverse correlation between $p_{\text {bar }}$ and $n$. This is an important point to note and might explain the contradictions between the results of previous studies, which found opposite trends of bar fraction with measures of bulge prominence from the light profile shape (e.g., Masters et al. 2011 compared to Barazza et al. 2008). Moreover, this observation is in good agreement with theoretical predictions of bar formation, as will be described in Section 4.

3. Central surface stellar mass density $\Sigma_{1 \mathrm{kpc}}^{*}($ Figure $3(c))$. We find similar trends of $p_{\text {bar }}$ with this parameter as between $p_{\text {bar }}$ and $n$. Star-forming galaxies show a correlation between $p_{\text {bar }}$ and $\Sigma_{1 \mathrm{kpc}}^{*}$ (star-forming disks are more likely to host bars where the central density is higher), while quiescent galaxies show an anticorrelation (quiescent disks are more likely to host bars where the central density is lower).

\subsection{Bar Length Trends}

In this section, we examine how BL depends on galaxy properties. We define a scaled BL $L_{\mathrm{sbar}}$ as the BL divided by a measure of disk size. We choose for this $2.2 r_{\mathrm{d}}$ (2.2 semimajor axis exponential disk scale lengths) because this is where the rotation curve of a self-gravitating exponential disk reaches its maximum (Freeman 1970). Hereafter, we will refer to the scaled BL simply as the BL unless stated otherwise.

Bars become longer over time as they transfer angular momentum from the bar to the outer disk and/or spheroid (halo and, whenever relevant, bulge). This secular evolution causes the host disk to expand and increase its scale length while the bar also grows. We will compare trends of BL with those of $p_{\text {bar }}$ to test if the trends we observe in the average value of $p_{\text {bar }}$ in the galaxy population are due to the evolution of the bars or the likelihood of bar formation in a galaxy.

Since the BL sample is more than an order of magnitude less numerous than the GZ2D sample, we find that breaking it up into small bins, as we did in Figure 3 for $p_{\text {bar }}$, results in no clear correlations. Since we found that the trends of $p_{\text {bar }}$ had different properties depending on the SSFR of the galaxies, we split the BL sample into two subsamples (star forming, or $\log$ SSFR $>-11 \mathrm{yr}^{-1}$, and quiescent, or $\log$ SSFR $<-11 \mathrm{yr}^{-1}$ ) to look at the trends of average $L_{\text {sbar }}$. These trends are shown in Figure 4.

Figure 4 shows that in the star-forming sequence, the average value of $L_{\text {sbar }}$ increases with all three properties $\left(M_{*}, n\right.$, and $\left.\Sigma_{1 \mathrm{kpc}}^{*}\right)$. In the quiescent population we find that the average BL decreases with $M_{*}$. Curiously, we find that the average BL increases with $n$ and $\Sigma_{1 \mathrm{kpc}}^{*}$ up to a maximum value at around $\log n \approx 0.6(n \approx 4)$ and $\Sigma_{1 \mathrm{kpc}}^{*} \approx 10^{9.8} M_{\odot} \mathrm{kpc}^{-2}$, respectively, where the trend reverses.

\section{COMPARISON TO THEORY}

In this section, we compare our results in Section 3 with theoretical expectations of bar formation and evolution. We start with a short summary of theoretical results.

\subsection{Theoretical Reminders}

One can distinguish (at least) two phases in the lifetime of a bar: the formation phase and the secular evolution phase. AMR13 showed that these two phases are contiguous in gas-rich cases, while for gas-poor ones they are not. In the latter 


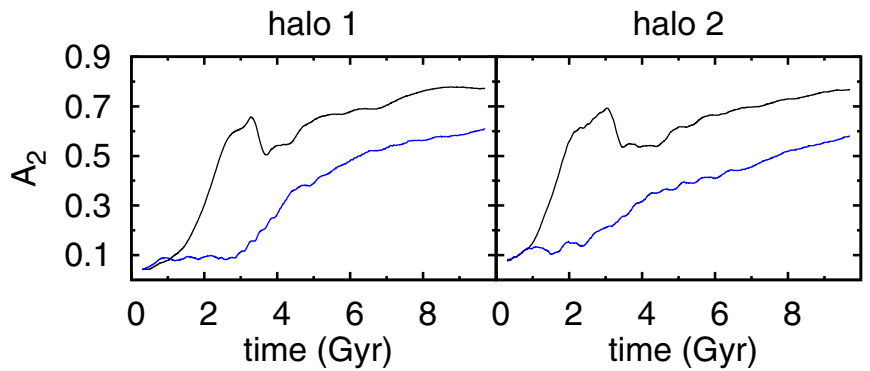

Figure 5. Bar strength $A_{2}$, which can considered as a rough proxy for bar length, as a function of time for four simulations. Two simulations have a gas-rich disk (blue lines), while the other two have a gas-poor disk (black lines). The two panels correspond to different types of haloes: (left) initially spherical and (right) initially triaxial. For a full description of these simulations and their results, see AMR13. These simulations show that bars grow slower and are less strong in the gas-rich case.

(A color version of this figure is available in the online journal.)

case, there are two further stages of relatively short duration in between the formation and secular evolution phases. ${ }^{22}$

This is illustrated in Figure 5, where we plot the bar strength $A_{2}$, which is closely related to $\mathrm{BL}$, as a function of time for four simulations from AMR13. The two simulations in each panel have the same initial mass and velocity distribution of the baryonic and dark matter components. The only difference is the gas fraction, where the black and blue lines represent gas-poor and gas-rich simulations, respectively. The end of the bar formation phase is represented by the time when the steep increase of $A_{2}$ terminates, which is at times of 2-2.5 Gyr for the gas-poor simulations and around $4.5 \mathrm{Gyr}$ for the gas-rich ones. These simulations illustrate that gas slows down bar formation considerably (AMR13). This is due to both an increase in the duration of the prebar phase (i.e., the phase during which the disk can still be considered as axisymmetric) and a decrease in the rate of the bar growth (i.e., an increase of the time it takes for the bar to end its growth phase), both being compared to the times of the equivalent phases in the gas-poor case.

The secular evolution phase, however, starts roughly at 4.5 Gyr for all cases. In general, the duration of these phases, as well as the increase of bar strength that they imply, depends on the mass and velocity distribution of the baryonic and dark matter components within the galaxy, as well as on the gas fraction. Readers can find more information and a long list of relevant references in a recent review by Athanassoula (2012). It is also interesting to note in Figure 5 that for all times and in both phases, the bar in the gas-rich case is less strong than in the gas-poor one (see also Berentzen et al. 2007).

Bar formation and evolution is influenced also by galactic bulges. Bulges, however, are an inhomogeneous class of objects (Kormendy 1993; Kormendy \& Kennicutt 2004; Athanassoula 2005). Classical bulges have high Sérsic indices, typically around 4 , but certainly above 2 . Disky pseudobulges, on the other hand, have low Sérsic indices, typically around 1 and usually less than 2 (Fisher \& Drory 2010). The most popular scenario for the formation of disky pseudobulges in barred galaxies is that they are due to stars and, particularly, gas pushed inward by the bar to the central parts of the disk. Here, the highdensity gas will give rise to star formation, so that the disky pseudobulges should be primarily composed of gas and young

\footnotetext{
22 These two extra stages are related to the bar buckling phase (i.e., the formation of a boxy/peanut bulge), which is much less obvious in gas-rich cases.
}

stars with a smaller fraction of old stars. Their extent is typically of the order of $1 \mathrm{kpc}$ (Athanassoula 1992; Heller \& Shlosman 1994; Fisher \& Drory 2010). ${ }^{23}$

These two different types of bulges have different dynamics and therefore different effects on the bar formation and evolution phases. Classical bulges predate the bar, so they will influence both phases. Their influence has many similarities to that of the dark matter halo. Namely, they slow down bar formation in the first phase, but during the secular evolution phase, they help the bar grow by absorbing angular momentum, leading to stronger bars (Athanassoula \& Misiriotis 2002, A03). Thus, simulations predict that bars in galaxies with classical bulges should be stronger than bars in galaxies without classical bulges, assuming all other properties are the same.

On the other hand, disky pseudobulges in barred galaxies are formed by material pushed inward by the bar; that is, they do not predate the bar and thus cannot influence its formation phase. Moreover, disky pseudobulges should not help the bar grow during the secular evolution phase either since they cannot absorb angular momentum. This is because the radii of disky pseudobulges are considerably smaller than the corotation radius and also because disky pseudobulges are flat (spherical-like density distributions, like the classical bulge or the halo, can absorb angular momentum). However, although disky pseudobulges do not affect bar formation or evolution, bars do affect disky pseudobulges. In fact, bar-driven secular evolution is the primary process of disky pseudobulge creation and growth (Kormendy \& Kennicutt 2004; Athanassoula 2005). Thus, the theoretical prediction is that stronger bars push more gas inward, resulting in more massive disky pseudobulges.

A visual approximation of bar formation and evolution is presented in Figure 6.

\subsection{Effect of Gas Content on Bar Formation}

We explain the trends we observe between the likelihood of disk galaxies being barred and their SSFR (present even at fixed $M_{*}, n$, or $\Sigma_{1 \mathrm{kpc}}^{*}$; see Figure 3 ) as being due to the effect of gas on bar formation. In the models, bars form later in disk galaxies with significant gas content, and after they form, they grow slower than disk galaxies with comparably less gas (AMR13 and Figure 5). This predicts that the bar likelihood should be higher in gas-poor galaxies (i.e., the quiescent population) simply because some of the gas-rich galaxies (i.e., the starforming sequence) have not yet formed their bars. Thus, taking SSFR as a tracer of gas content, there is good agreement between simulation results and the trends we find (see also Masters et al. 2012).

Within the star-forming sequence (defined here as $\log$ SSFR $>-11 \mathrm{yr}^{-1}$ ) disk galaxies do not all have the same $p_{\text {bar }}$, but neither do they all have the same gas content. There are well-known trends between SSFR, stellar mass, and gas content of disk galaxies (e.g., Catinella et al. 2010; Saintonge et al. 2011). The trend we observe here for $p_{\text {bar }}$ to increase as SSFR declines (and $M_{*}$ increases) can be explained as being due to decreasing amounts of gas in the disks of these galaxies. Indeed, Masters et al. (2012) showed that if you correct for the typical

\footnotetext{
23 For completeness, we mention the boxy/peanut bulges, which are, in fact, part of the bar. Their Sérsic indices are smaller than or of the order of that of the disky pseudobulges. Given that all our decompositions here include only one or two components (Section 2.3) and bars are not included and our sample excludes highly inclined systems, such bulges do not enter in our discussion. However, we do note that they may still be present in the sample and may not be well fit by our decompositions.
} 


\section{Schematic of Bar Formation and Evolution}
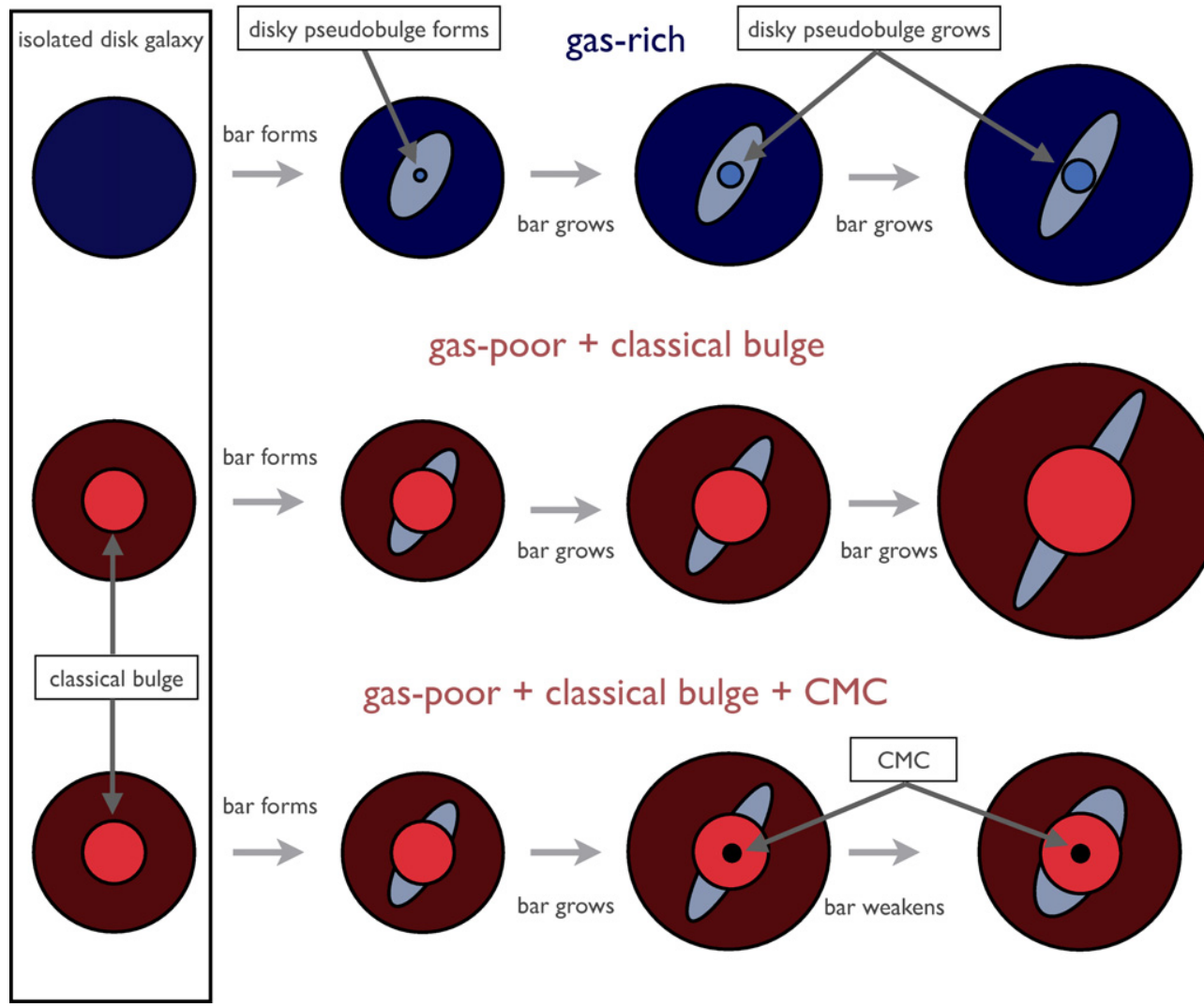

gas-poor + classical bulge

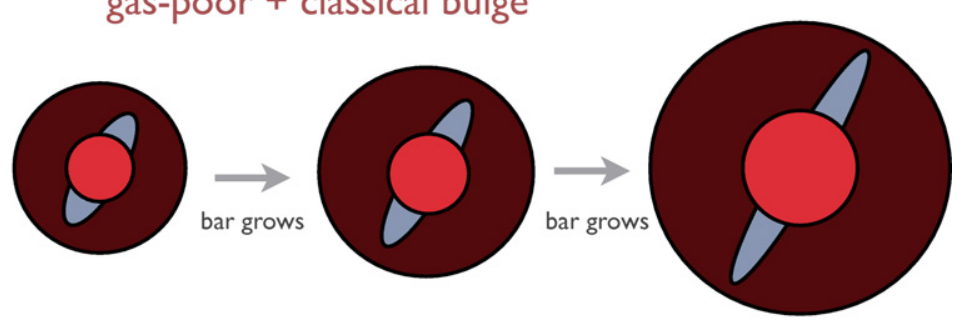

gas-poor + classical bulge + CMC
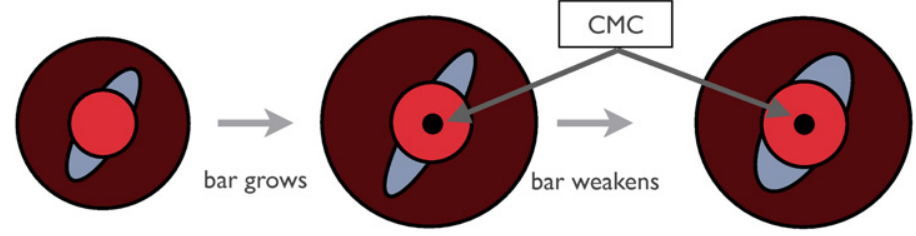

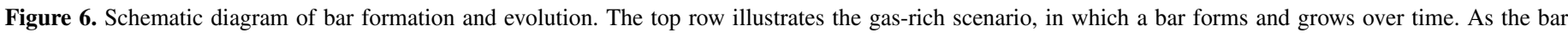

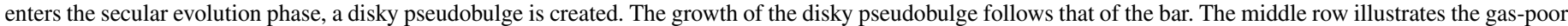

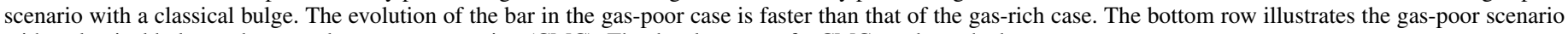
with a classical bulge and a central mass concentration (CMC). The development of a CMC weakens the bar.

(A color version of this figure is available in the online journal.)

H I content of a disk galaxy, those galaxies with more $\mathrm{H}_{\mathrm{I}}$ than is expected for their stellar mass are less likely to host bars.

\subsection{Effects of Classical Bulges and Disky Pseudobulges on Bar Formation}

We observe trends of bar likelihood with the Sérsic index ( $n$, Figure 3(b)) and central surface stellar mass density $\left(\Sigma_{1 \mathrm{kpc}}^{*}\right.$, Figure 3(c)), where the latter two parameters are considered to be measures of bulge prominence. In the star-forming sequence bar likelihood increases with both increasing $n$ and $\Sigma_{1 \mathrm{kpc}}^{*}$, while the opposite trend is observed in the quiescent population. In order to interpret these trends, we need to remember that there are two main types of bulges, the classical bulge and the disky pseudobulge, a distinction that will help explain this dichotomy.

The best way to distinguish these types of bulges involves the use of high-resolution imaging of the bulges (e.g., Fisher \& Drory 2008), something that is not available for our large sample. However, one can approximately separate the types with a threshold in the global galaxy Sérsic index (Drory \& Fisher 2007). ${ }^{24}$ Disky pseudobulges generally lie in galaxies

\footnotetext{
24 Gadotti (2009) advocates using the Kormendy relationship to separate classical bulges from disky pseudobulges. For this work, however, we choose to use the more simple global galaxy Sérsic threshold.
}

with global $n<2.5$, while classical bulges are found in galaxies with global $n>2.5$. Although this method is less accurate than those using high-resolution imaging, this is a simple option that is adequate for our purposes. Hence, we adopt this Sérsic threshold for the rest of the paper to distinguish the two types of bulges. ${ }^{25}$ This threshold is illustrated with a vertical dashed line at $\log n=0.4(n=2.5)$ in Figure 3(b).

Our sample confirms the well-known observation (e.g., Drory $\&$ Fisher 2007) that quiescent (red) disk galaxies primarily have classical bulges, while star-forming (blue) disk galaxies mainly have disky pseudobulges (see Figure 3(b)). This suggests that the decreasing $p_{\text {bar }}$ with $n$ and $\Sigma_{1 \mathrm{kpc}}^{*}$ observed in the quiescent disk galaxies is due to $p_{\text {bar }}$ decreasing in galaxies with larger classical bulges, while the increasing $p_{\text {bar }}$ with $n$ and $\Sigma_{1 \mathrm{kpc}}^{*}$ observed in star-forming disks shows that $p_{\text {bar }}$ is larger in galaxies with more massive disky pseudobulges.

The classical bulge, like the halo, slows down bar formation because it "dilutes" the nonaxisymmetric forcing of the bar (Athanassoula 2012). This predicts that bar likelihood should

\footnotetext{
25 Of course, there are $n<2.5$ galaxies that have no bulge (e.g., Simmons et al. 2013). However, for simplicity, we consider all galaxies with $n<2.5$ to contain a disky pseudobulge even if it might be a pure disk galaxy. This will not affect our discussion since pure disks and disky pseudobulges are closely related (see Kormendy \& Kennicutt 2004).
} 

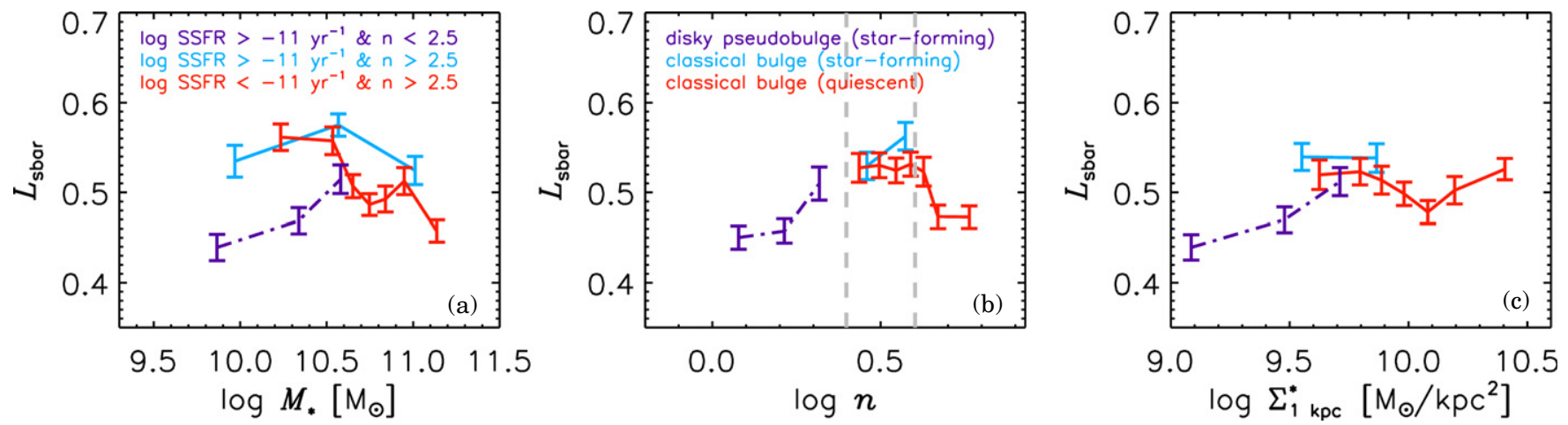

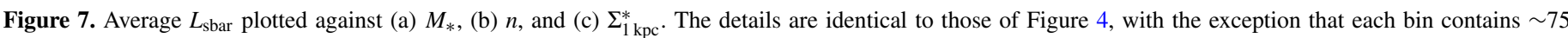

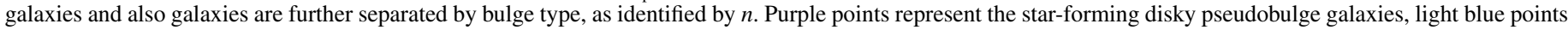

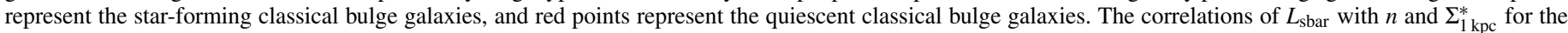
disky pseudobulge galaxies match the predictions of bar-driven secular evolution.

(A color version of this figure is available in the online journal.)

decrease with increasing prominence of the classical bulge, as we indeed observe.

Disky pseudobulges result from the material that a bar pushes inward to the central part of the disk. Since these bulges formed after the bar and are, in fact, a product of the bar, they cannot influence the bar formation phase. However, there is a clear link predicted between the existence of the bar and the amount of mass in the disky pseudobulge (or central $1 \mathrm{kpc}$; Athanassoula 1992; Heller \& Shlosman 1994; Fisher \& Drory 2010). For galaxies of a given gas mass (or SSFR), a higher bar likelihood should result in more massive disky pseudobulges, as we observe (Figures 3(b) and (c)).

\subsection{Evidence for Secular Evolution}

BL trends (Section 3.2) can help us understand the secular evolution phase of the bar. We can safely assume that during the secular evolution phase of any noninteracting galaxy its BL may be considered a proxy of bar age. However, this may not be true for any two galaxies because the galaxy with the youngest bar can have the longest bar, provided its halo can absorb larger parts of the angular momentum emitted by the bar region (A03). Our comparisons, however, do not concern two galaxies but ensembles of a relatively large number of galaxies. For example, in Figure 4(b), we compare ensembles of galaxies with different $n$ values. But the number of galaxies in each ensemble is sufficiently large for us to assume that galaxies with a variety of halo properties are included in a roughly similar manner in all ensembles. This subtle, but important, point is intrinsic in our analysis and will be discussed further in Section 6.

The strongest $L_{\text {sbar }}$ trends we observe are found within starforming disk galaxies (the average $L_{\text {sbar }}$ increases monotonically with $M_{*}, n$, and $\Sigma_{1 \mathrm{kpc}}^{*}$; see Figure 4$)$. To better understand the underlying physical processes responsible for these trends, we separate the data in Figure 4 by bulge type; this is shown in Figure 7. Recall that galaxies with $n<2.5$ are considered to contain disky pseudobulges, while galaxies with $n>2.5$ are considered to contain classical bulges. Note that quiescent galaxies with $n<2.5$ are very rare; hence, they are not shown.

During the secular evolution phase, bars become stronger, longer, and more efficient at funneling gas into the central regions of galaxies, leading to more massive disky pseudobulges (AMR13). This prediction matches our observations in Figures $7(\mathrm{~b})$ and (c), where it is clear that $L_{\mathrm{sbar}}$ is correlated with $n$ and $\Sigma_{1 \mathrm{kpc}}^{*}$ for the disky pseudobulge galaxies (purple). These correlations give evidence for the secular evolution phase of bars.

The $L_{\mathrm{sbar}}$ trends with classical bulges are much more complex and also much less straightforward to interpret. Simulations show that classical bulges should foster secular evolution by absorbing some of the angular momentum emitted by the bar region (A03). Hence, the expectation is that galaxies with more massive classical bulges should have longer bars and also longer disk scale lengths.

Figures 7(b) and (c) show that the classical bulge galaxies (light blue and red) generally have longer scaled BLs than galaxies without a classical bulge, i.e., the disky pseudobulge galaxies. However, there is little evidence of increasing scaled BL with increasing $n$ and $\Sigma_{1 \mathrm{kpc}}^{*}$. In fact, there actually appears to be a decrease in scaled BL for $\log n$ larger than 0.6 (i.e., $n$ larger than about 4 , equivalent to a more concentrated light profile than the standard $r^{1 / 4}$ de Vaucouleurs profile). Similarly, the scaled BL stops increasing in the rightmost panel for $\Sigma_{1 \mathrm{kpc}}^{*}$ larger than roughly $10^{9.8} M_{\odot} \mathrm{kpc}^{-2}$ (although there seems to be a final increase in scaled BL at the highest $\Sigma_{1 \mathrm{kpc}}^{*}$ ).

This decrease in scaled BL with large $n$ and $\Sigma_{1 \mathrm{kpc}}^{*}$ does not disagree with simulation results and can be attributed to the presence of a very high central mass concentration (CMC). ${ }^{26}$ Indeed, our last averaged point is roughly at a $\log n$ value of 0.8 , which corresponds to a Sérsic index of roughly 6.5 . This could well be due to a luminosity spike in the center of the galaxy, which would hamper the bar growth and evolution if it preexisted the bar or, if grown later, which would bring a decrease of the BL and strength (e.g., Shen \& Sellwood 2004; Athanassoula et al. 2005). This strong CMC will thus bring a decrease of BL at the highest values of $n$, as seen in Figure 7(b) (and Figure 4(b)).

Nevertheless, at least part of this decrease could be spurious and because the bar component is not specifically included in our two-component decompositions, which is more worrisome for galaxies with stronger and longer bars. To test the possibility that the disk scale length, which comes from the GIM2D bulge+disk decompositions, may be unreliable, we scaled the absolute $\mathrm{BL}$

\footnotetext{
26 We note that this is just one of the possible reasons for this observed decrease. This could also correspond to the regime where the bulge is so massive that it has significantly delayed the onset of bar formation, resulting in a lack of bar evolution.
} 
with the $r$-band isophotal radius at $25 \mathrm{mag} \operatorname{arcsec}^{-2}$ from the SDSS pipeline and recreated Figures 4 and 7 . The results can be found in Appendix B. We find then that the decrease seen with the disk scale length at high $n$ and $\Sigma_{1 \mathrm{kpc}}^{*}$ is considerably lessened. We do not fully understand the BL trends with the classical bulge galaxies at the highest $n$ and $\Sigma_{1 \mathrm{kpc}}^{*}$; more work needs to be done. Let us note, however, that the correlations of the scaled BL with the disky pseudobulge galaxies are still present even when scaling with the isophotal radius, thus enhancing our confidence in the corresponding decompositions and trends.

\section{DISCUSSION}

\subsection{Are We Observing Secular Evolution?}

In Sections 3 and 4, we showed evidence that suggests that disky pseudobulges are more massive in populations of galaxies that are more likely to host bars and that host longer bars (specifically, that average values of $p_{\text {bar }}$ and $L_{\text {sbar }}$ increased with $n$ and $\Sigma_{1 \mathrm{kpc}}^{*}$ for disky pseudobulge galaxies). We interpreted this as observational evidence of bar-driven secular evolution growing disky pseudobulges (Kormendy \& Kennicutt 2004; Athanassoula 2005). Our interpretation hinges on the assumption that BL traces the evolution of bars. This assumption is based on both simulations of bar growth and observational data. Elmegreen et al. (2007) showed that BL mirrors bar strength (see also Block et al. 2004). The simulations of bar growth shown in Figure 5-and a large number of others, as reviewed by Athanassoula (2012)—demonstrate that isolated bars typically grow stronger with time.

Furthermore, the simulations of AMR13 argue that bars in isolated galaxies are long-lived structures: in the $\sim 10 \mathrm{Gyr}$ that their simulations covered, not one of their bars dissolved (see also Debattista et al. 2006 and Berentzen et al. 2007 for a similar conclusion). Recent zoom-in cosmological simulations by Kraljic et al. (2012) also support the idea that bars are long-lived structures. Their simulations show that most of the bars that formed at $z \leqslant 1$, when mergers become less frequent, persist down to $z=0$. Observational studies have now observed bars with modest frequencies out to $z \sim 1$ (Abraham et al. 1999; Jogee et al. 2004; Elmegreen et al. 2004; Sheth et al. 2008; Cameron et al. 2010; Melvin et al. 2013), and one upcoming study detects bars as far out as $z \sim 1.5$ (Herrington et al. 2013), with the implication that many of the bars we observe in the local universe could have formed at $z \sim 1$ or earlier. This gives a substantial time window for secular evolution to grow longer bars and stronger disky pseudobulges.

Previous works have shown a trend between BL and Hubble type - that bars are longer in earlier type disks - and have used this to argue that secular evolution had been observed (e.g., Athanassoula \& Martinet 1980; Elmegreen \& Elmegreen 1985; Martin 1995; Regan \& Elmegreen 1997; Erwin 2005; Laurikainen et al. 2007; Elmegreen et al. 2007; MenéndezDelmestre et al. 2007; Gadotti 2011). Our result is novel in that it looked for trends of BL with the central mass density in the very centers of galaxies, a quantity that is directly linked to secular evolution in models. Our sample is also nearly an order of magnitude larger than any previous study. Thus, we argue that our result is the best evidence yet for bar-driven secular evolution in disk galaxies.

Recent results from several high-resolution simulations present mechanisms for the formation of disky pseudobulges that do not rely on secular evolution (Inoue \& Saitoh 2012; Okamoto 2013), but rather involve dynamical instability in clumpy galaxies or high-redshift starbursts. While the bulges of these simulations do have characteristics of local disky pseudobulges, ${ }^{27}$ our results here suggest that secular evolution does have a major effect, both in creating disky pseudobulges and in building up the stellar mass in the bulge region of barred galaxies.

Nevertheless, there are substantial numbers of disk galaxies that are nonbarred and are hosting disky pseudobulges (Kormendy \& Kennicutt 2004). Up to a third or more of the local disk galaxy population is unbarred in even the most conservative reckoning. This observations argues that disky pseudobulges have more than one formation mechanism. Perhaps disky pseudobulges in nonbarred galaxies were created through high-redshift channels, while the disky pseudobulges in barred galaxies may have been created and are still in the process of growing through bar-driven secular evolution at much later times.

\subsection{Can Bars Quench Star Formation?}

The highest values of $p_{\text {bar }}$ are found among quiescent galaxies with $n \sim 2.5$ (see Figure 3(b)). Here we consider the question of whether these bars were formed in situ or if they could be implicated in the processes that turned these disk galaxies quiescent. We ask, "Were these bars formed in $n \sim 2.5$ quiescent galaxies, or did they form in star-forming galaxies (with $n \lesssim 2.5$ ) that evolved into the $n \sim 2.5$ quiescent disk galaxies?" We refer to this latter process as "bar quenching" and explore this idea further.

Bars have been associated with enhanced central star formation in galaxies for decades (Hawarden et al. 1986; Dressel 1988; Friedli \& Benz 1993; Giuricin et al. 1994; Huang et al. 1996; Martinet \& Friedli 1997; Martin \& Friedli 1997; Ho et al. 1997; Ellison et al. 2011; Oh et al. 2012; Wang et al. 2012). This is a natural consequence of the evolution of gas in a disk galaxy under the influence of a bar. The bar-induced gravitational torques funnel gas into the centers of galaxies (Matsuda \& Nelson 1977; Simkin et al. 1980; Athanassoula 1992; Wada \& Habe 1992, 1995; Friedli \& Benz 1993; Heller \& Shlosman 1994; Knapen et al. 1995; Sakamoto et al. 1999; Sheth et al. 2005), where it should quickly form stars, thus enhancing the central star formation. If this secular evolution were efficient, it could accelerate the depletion of the gas supply within a considerable fraction of the disk, namely, the region within corotation. If this process were not balanced by an increased inflow of cosmological gas, this would ultimately produce a quiescent barred galaxy (Masters et al. 2011; Masters et al. 2012).

Large surveys such as SDSS (Abazajian et al. 2009), the Cosmic Evolution Survey (Scoville et al. 2007), and the AllWavelength Extended Groth Strip International Survey (Davis et al. 2007) have painted a clear picture of the structural properties of quiescent galaxies: they are massive, centrally concentrated, and have high central velocity dispersions (e.g., Franx et al. 2008; Bell et al. 2012; Wake et al. 2012; Cheung et al. 2012; Fang et al. 2013; Williams et al. 2013; Barro et al. 2013). Cheung et al. (2012) recently found that the most distinguishing structural parameter of quiescent galaxies (compared to starforming galaxies) is their central surface stellar mass density (within a radius of $1 \mathrm{kpc}$ ). Almost all quiescent galaxies in the sample of Cheung et al. (2012) have high values of $\Sigma_{1 \mathrm{kpc}}^{*}$, while

\footnotetext{
27 Not all bulges made from clump coalescence have characteristics of disky pseudobulges. For example, Elmegreen et al. (2008) show that their bulges made through clump coalescence have properties of classical bulges.
} 
star-forming galaxies mainly have low values of $\Sigma_{1 \mathrm{kpc}}^{*}$. This is clear evidence that the process(es) that quenches star formation in these galaxies is related to the buildup of the central stellar mass density (see also Fang et al. 2013). We consider here if secular evolution is able to build high enough central densities to act as a quenching mechanism.

Indeed, Figure 7(c) shows that the $\Sigma_{1 \mathrm{kpc}}^{*}$ values of the disky pseudobulge galaxies overlap partly with the $\Sigma_{1 \mathrm{kpc}}^{*}$ values of the classical bulge galaxies. The most massive of the disky pseudobulges that we argue are grown by bar-driven secular evolution are comparable in central density to the smallest of the classical bulges. This suggests that secular evolution can build the high central densities that are observed in quiescent galaxies. This appears to be circumstantial evidence for an interesting, and potentially important, galaxy evolution process: bar quenching. We caution, however, that our identification of disky pseudobulges in quiescent barred disk galaxies is based on global Sérsic fits. If it is indeed the case that there exist quiescent disk galaxies that host only disky pseudobulges and show no evidence for classical bulges, this will be strong evidence of the process of "bar quenching" having acted in these galaxies. However, more accurate identifications of disky pseudobulges are needed to verify this claim.

\section{CONCLUSION}

In this paper, we use hundreds of thousands of visual classification measurements of galactic bars provided by citizen scientists through the GZ project (Lintott et al. 2008, 2011; Willett et al. 2013). We first select a sample of disk galaxies in which reliable bar classifications can be made; we call this the GZ2D sample, which comprises 13,295 oblique (i.e., face-on or mildly inclined) disk galaxies in a volume limit to $z=0.06$. This sample is similar to the GZ2 samples used previously to study trends of the bar fraction by Masters et al. (2011) and Masters et al. (2012). Strongly barred galaxies identified in GZ2 were part of a small GZ project that used a Google Sky interface to collect measurements of BLs (Hoyle et al. 2011). In this paper we also make use of this bar length sample, which comprises 1154 galaxies. We use these data to analyze the dependence of bar likelihood ( $p_{\text {bar }}$, a weighted and debiased fraction of GZ users identifying a bar, which acts like the probability of a galaxy containing a visually identifiable bar; Willett et al. 2013) and scaled BL $\left(L_{\mathrm{sbar}}=L_{\mathrm{bar}} / 2.2 r_{\mathrm{d}}\right.$; a measure of bar strength, linked to how evolved a bar is) on other galactic properties. Specifically, we test how the likelihood and length of bars depend on the SSFR (estimated through nebular emission lines from the SDSS fiber and the broadband ugriz SDSS photometry, as measured by MPA-JHU) and inner galactic structure (i.e., bulge prominence) parameterized by the global Sérsic index $n$, as measured by GIM2D, and the central surface stellar mass density $\Sigma_{1 \mathrm{kpc}}^{*}$, as estimated from a $1 \mathrm{kpc}$ radius circular aperture projected onto SDSS images.

Our main observational results (Section 3) are as follows.

1. There exists an anticorrelation between $p_{\text {bar }}$ and SSFR; this relationship is present even at fixed $M_{*}, n$, or $\Sigma_{1 \mathrm{kpc}}^{*}$.

2. The structural trends of $p_{\text {bar }}$ are bimodal with SSFR. In the star-forming sequence, $p_{\text {bar }}$ correlates with $n$ and $\Sigma_{1 \mathrm{kpc}}^{*}$, while in the quiescent population, $p_{\text {bar }}$ anticorrelates with $n$ and $\Sigma_{1 \mathrm{kpc}}^{*}$.

3. The structural trends of $L_{\text {sbar }}$ are also bimodal with SSFR. Within the star-forming sequence, $L_{\text {sbar }}$ correlates with $n$ and $\Sigma_{1 \mathrm{kpc}}^{*}$, in a similar way to $p_{\mathrm{bar}}$. However, within the quiescent population, $L_{\text {sbar }}$ shows a rather different behavior, with a peak at values of $n \sim 4$ and $\Sigma_{1 \mathrm{kpc}}^{*} \sim$ $10^{9.8} M_{\odot} \mathrm{kpc}^{-2}$.

We compare these results to simulations of bar formation and evolution in Section 4. We find that the underlying physical processes become clearer upon separating these galaxies by those that contain disky pseudobulges $(n<2.5)$ and those that contain classical bulges $(n>2.5)$. This comparison reveals the following.

1. Assuming that SSFR is a good tracer of gas content, the anticorrelation of $p_{\text {bar }}$ with SSFR is consistent with the expected effects of gas on bar formation. Simulations show that gas delays the formation of bars; thus, many gasrich galaxies simply have not yet formed bars, while most gas-poor disk galaxies have.

2. The observed trends of $p_{\mathrm{bar}}$ and $L_{\mathrm{sbar}}$ with $n$ and $\Sigma_{1 \mathrm{kpc}}^{*}$ for classical bulge galaxies are consistent with the effects of classical bulges and CMCs on bar formation and evolution. The gravitational forcing of classical bulges dilute the nonaxisymmetric forcing of the bar, which delays the formation of a bar. This diluting effect is more powerful in more massive classical bulges, resulting in a longer delay of bar formation. After the bar has formed, however, classical bulges are expected to promote secular evolution by absorbing the angular momentum emitted from the bar region; this process also scales with the mass of the bulge and leads to both longer bars and longer disk scale lengths. Our results suggest that for a Sérsic index up to roughly $n=4$ the BL may increase faster than the disk scale length. For yet higher values of $n$, a strong ensuing CMC could lead to a decrease of the bar strength by generating instabilities of the main family of bar-supporting orbits.

3. The correlations of $p_{\text {bar }}$ and $L_{\text {sbar }}$ with $n$ and $\Sigma_{1 \mathrm{kpc}}^{*}$ for the disky pseudobulge galaxies are in agreement with the predictions of bar-driven secular evolution. Bars drive gas toward the centers of galaxies, where the gas should eventually form stars and give rise to disky pseudobulges. As bars grow stronger and longer, the ability to funnel gas grows stronger as well, resulting in more massive disky pseudobulges.

The comparison of the observational results we present here with simulations of bar formation and growth shows general agreement, indicating that many of the underlying physical processes of bar formation and evolution are understood. An implication of this is that we are confident in our basic understanding of the relationship between bars and their host galaxies. Bars are clearly not stagnant structures; rather, they are dynamic and evolving and, furthermore, directly influence the evolution of their host galaxies.

Although this work only concerns the universe at $z \sim 0$, the ramifications of this idea reach far beyond the local universe. There is increasing evidence that bars have been present since $z \sim 1$ (Abraham et al. 1999; Jogee et al. 2004; Elmegreen et al. 2004; Sheth et al. 2008; Cameron et al. 2010; Melvin et al. 2013; Herrington et al. 2013), indicating that the evolution of disk galaxies has been affected by bars for the last $\sim 8$ billion years. Moreover, if the observed evolution of bar fraction with redshift is extrapolated into the future (there is now an agreement that bar fraction increases toward lower $z$ ), then bars will soon be present in nearly all disk galaxies and hence will become an even more dominant driver of disk galaxy evolution. 
We cannot yet claim to understand all aspects of the symbiotic relationship between bars and their host galaxies. We do not fully understand the complicated behavior we observe between BL and inner galactic structure in disk galaxies hosting classical bulges. Our tentative explanation is that these trends are due to the presence of CMCs; however, this should be tested with much higher resolution imaging to probe the very centers of galaxies. Furthermore, in this work we have not explored many of the parameters that are predicted to affect bar formation and evolution (e.g., the dark matter halo and the velocity dispersion of the stars in the disk; Athanassoula \& Sellwood 1986; A03). Even so, we found a good agreement between theory and observations, and all observational trends could be well explained by simulations. This may be due to the large size of our sample, which allows for a variety of halo properties and of disk velocity dispersions in a roughly similar manner in all ensembles we compared. Finally, the role bars may play in processes that quench star formation is an interesting and potentially important issue for galaxy evolution that warrants further study.

The most notable success in our comparison between observation and theory is the evidence we present for secular evolution. Unlike galaxy mergers, secular evolution is a slow and gentle process that is not immediately obvious in images. There has been previous observational evidence of secular evolution in galaxies (e.g., Athanassoula \& Martinet 1980; Courteau et al. 1996; MacArthur et al. 2003; Elmegreen et al. 2007; Laurikainen et al. 2009; Coelho \& Gadotti 2011; Sánchez-Janssen \& Gadotti 2013); however, the combination of our large data set and the observed correlations of bar likelihood and BL with inner galactic structure for star-forming disk galaxies makes our results one of the most compelling pieces of evidence of not only the existence of secular evolution but also the role of ongoing secular processes in the evolution of disk galaxies.

This publication has been made possible by the participation of more than 200,000 volunteers in the GZ project. Their contributions are individually acknowledged at http://www.galaxyzoo.org/Volunteers.aspx.

E.C. acknowledges financial support from the National Science Foundation grant AST 08-08133. E.A. and A.B. acknowledge financial support to the DAGAL network from the People Programme (Marie Curie Actions) of the European Union's Seventh Framework Programme FP7/2007-2013/ under REA grant agreement number PITN-GA-2011-289313. They also acknowledge financial support from the CNES (Centre National d'Etudes Spatiales, France). K.L.M. acknowledges funding from The Leverhulme Trust as a 2010 Early Career Fellow. R.C.N. acknowledges STFC Rolling grant ST/I001204/ 1 "Survey Cosmology and Astrophysics." K.S. gratefully acknowledges support from Swiss National Science Foundation grant PP00P2_138979/1. R.A.S. is supported by NSF grant AST-1055081. K.W.W. is supported by the U.S. National Science Foundation under grant DRL-0941610. E.A., E.B., and A.B. thank the Aspen Center for Physics for their hospitality during the workshop "The Milky Way as a Laboratory for Galaxy Formation" in 2013 and the NSF for partial financial support. Part of the simulation work described here was performed using HPC resources from GENCI-TGCC/CINES (grant 2013x2013047098).

Funding for SDSS and SDSS-II has been provided by the Alfred P. Sloan Foundation, the Participating Institutions, the National Science Foundation, the U.S. Department of Energy,
NASA, the Japanese Monbukagakusho, the Max Planck Society, and the Higher Education Funding Council for England. The SDSS Web site is http://www.sdss.org/. The SDSS is managed by the Astrophysical Research Consortium for the Participating Institutions. The Participating Institutions are the American Museum of Natural History, Astrophysical Institute Potsdam, University of Basel, University of Cambridge, Case Western Reserve University, University of Chicago, Drexel University, Fermilab, the Institute for Advanced Study, the Japan Participation Group, Johns Hopkins University, the Joint Institute for Nuclear Astrophysics, the Kavli Institute for Particle Astrophysics and Cosmology, the Korean Scientist Group, the Chinese Academy of Sciences (LAMOST), Los Alamos National Laboratory, the Max Planck Institute for Astronomy (MPIA), the Max Planck Institute for Astrophysics (MPA), New Mexico State University, Ohio State University, University of Pittsburgh, University of Portsmouth, Princeton University, the U.S. Naval Observatory, and the University of Washington.

E.C. thanks Charlie Conroy, Jonathan R. Trump, Guillermo Barro, Jerome Fang, and Yicheng Guo for useful discussions, comments, and suggestions. We also thank the anonymous referee for a very helpful report that resulted in substantial improvements to the paper.

\section{APPENDIX A COMPLETENESS}

\section{A.1. Galaxy Zoo 2 Disk Sample}

The GZ2D sample was selected on the basis of hundreds of thousands of visual morphological classifications collected via the GZ Web site. In order for a galaxy to be in this sample, the majority of volunteers classifying it must have identified "features" in it and identified it as not being an edge-on disk (see Masters et al. 2011; Willett et al. 2013). In addition, we apply an extra cut at $b / a>0.5$ to ensure disks are face-on enough to identify bars. This results in a sample of disk galaxies with a broad mix of Hubble types. Objects might contain an obvious disk (e.g., SBc, Sc) or a subtle disk (e.g., S0). While we do not expect problems in identifying the former in any orientation, S0 galaxies are notoriously difficult (even for the most expert classifiers) to separate from ellipticals if viewed face-on. We consider in this section if any face-on disk galaxies are missing from our sample. Presumably, if a galaxy had a bar, it would be readily identify as "featured" and included in this sample; thus, we assume any missing disk galaxies will be nonbarred and therefore introduce potential biases into our results.

We use as a comparison sample, the volume-limited parent GZ2 sample (see Section 2.2 for details of our initial GZ2 sample) that the GZ2D sample was originally drawn from, as well as a sample of edge-on disk galaxies in which we expect all types of disks will be equally easy to identify. As a reminder, the parent GZ2 sample has the following criteria: (1) $0.01<z<0.06$, where $z$ is the SDSS spectroscopic redshift; and (2) $M_{r} \leqslant-20.15$, where $M_{r}$ is the rest-frame absolute Petrosian $r$-band magnitude.

Hereafter, this sample will be referred to as the GZ2 sample. We match the GZ2 sample to the MPA-JHU catalog for stellar masses and SFRs, resulting in a total of 43,221 galaxies.

To identify edge-on disks, we use thresholds in the GZ vote fractions for "features of disk" $\left(p_{\text {features }}>0.5\right)$ and for "edge-on disk" ( $\left.p_{\text {edge-on }}>0.80\right)$; this is slightly more conservative than the recommended thresholds for selecting a "clean edge-on" sample as given in Willett et al. (2013), but we do not expect 

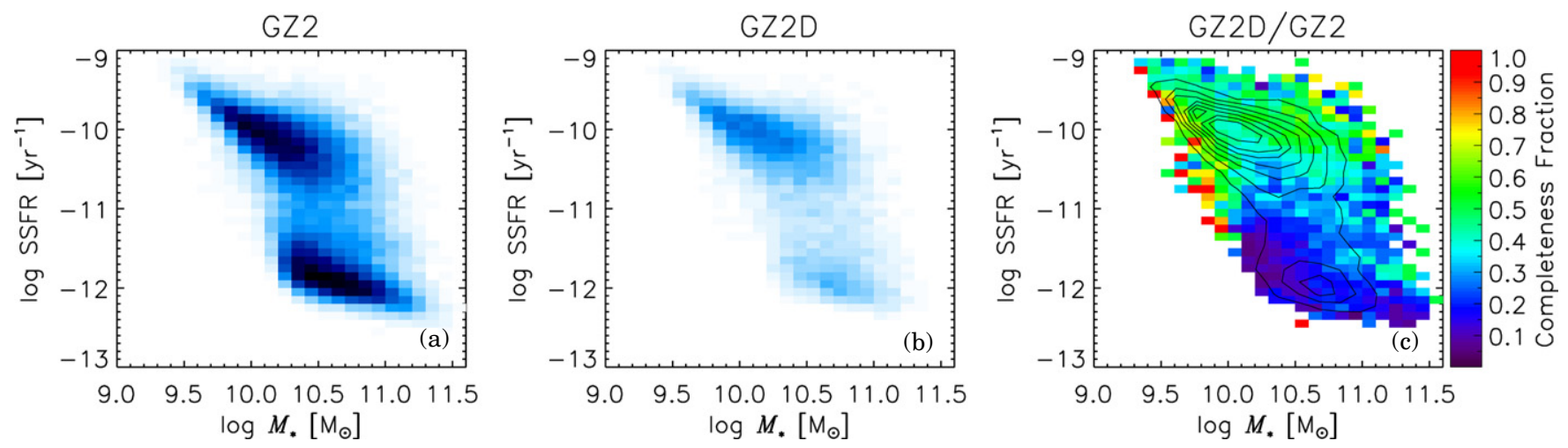

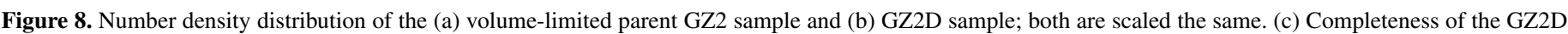

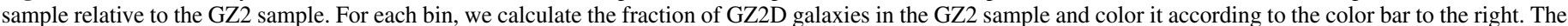

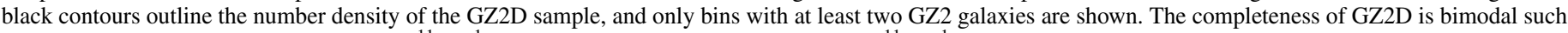
that it recovers $\sim 50 \%$ of high-SSFR $\left(>10^{-11} \mathrm{yr}^{-1}\right)$ galaxies and $\sim 20 \%$ of low-SSFR $\left(<10^{-11} \mathrm{yr}^{-1}\right) \mathrm{galaxies}$.

(A color version of this figure is available in the online journal.)

the selection to introduce any bias with Hubble type for disk galaxies.

Figure 8 compares the number density distribution of the GZ2D sample (panel (b)) to that of the whole volume-limited GZ2 sample (panel (a)). Both panels are scaled so that the blue scale indicates the same range of density and only bins with at least two galaxies are shown. Using the GZ2 sample as the fiducial completeness standard, panel (c) displays the completeness of the GZ2D sample (i.e., the fraction of the GZ2 sample that is in GZ2D) as indicated by the legend. To aid the eye, contours of the GZ2D number density distribution are overlaid. We point out that completeness levels of greater than $50 \%$ are not expected since the selection on axial ratio $(b / a>0.5)$ removes approximately half of all disk galaxies. In this plot we observe the expected bimodality, such that the completeness of high-SSFR ( $>10^{-11} \mathrm{yr}^{-1}$; "star-forming") galaxies is much higher $(\approx 50 \%$ complete $)$ than it is for lowSSFR $\left(<10^{-11} \mathrm{yr}^{-1}\right.$; quiescent) galaxies $(\approx 20 \%$ complete $)$. This reveals the well-known correlation between SSFR and morphology that most star-forming galaxies have disks and many quiescent galaxies are elliptical and so do not have obvious features to be selected as part of the GZ2D sample.

This test, however, cannot reveal if the GZ2D sample represents a fair selection of all disks. To test that, we isolate a sample of edge-on disk galaxies in which we expect all disks (even S0s) will be identified. If the GZ2D sample is fairly representative of all disk galaxies, then the ratio of GZ2D (face-on disk) galaxies to the sample of edge-on disks should be uniform throughout the SSFR-mass diagram (this assumes all disk galaxies are randomly orientated, which we expect they should be, but also see Simard et al. 2011, and that the inclination introduces no systematic biases into estimates of SSFR or stellar mass, which is less clear).

Figure 9 compares the number density of the edge-on GZ2 disks to our GZ2D sample of mildly inclined or face-on disk galaxies. We show the ratio of the number of galaxies in the GZ2D sample to the edge-on GZ2 disk sample. Only bins with at least two galaxies from the edge-on GZ2 disk sample are shown, and the black contours represent the number density distribution of the GZ2D sample. For high-SSFR galaxies, there are about seven galaxies in the GZ2D sample for every edgeon GZ2 disk galaxy. This is likely due to a combination of the expected number ratios for edge-on and non-edge-on disk

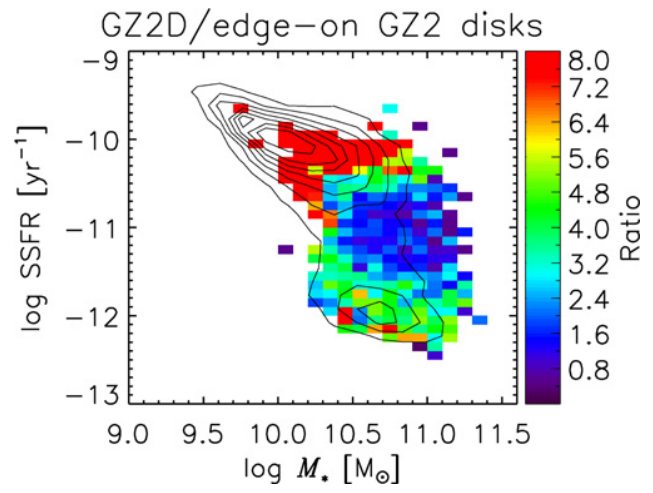

Figure 9. Ratio of the number of galaxies in the GZ2D sample to the edge-on GZ2 disk sample. The black contours outline the number density distribution of the GZ2D sample. Only bins with $n \geqslant 2$ edge-on GZ2 galaxies are shown. GZ2D does not seem to be strongly biased against low-mass quiescent disks.

(A color version of this figure is available in the online journal.)

galaxies (e.g., for random orientations, we expect one galaxy with $i>85^{\circ}$ for every five with $i<65^{\circ}$ ) and the possible effects of increased internal extinction in the edge-on sample causing SSFR to be underestimated. However, we do not expect to miss systematically any star-forming disk galaxies.

Because of the extinction of edge-on galaxies, the sample of low-SSFR ( $\log$ SSFR $\left.<-11.6 \mathrm{yr}^{-1}\right)$ edge-on GZ2 disks may contain a combination of truly low-SSFR disks and reddened intermediate-SSFR disks. However, we assume that the reddened intermediate-SSFR contribution to the low-SSFR regime of the edge-on GZ2 disk sample is uniform across stellar mass and changes only the absolute scaling of the number ratio between GZ2D and edge-on GZ2 disks. We therefore examine the uniformity of the low-SSFR regime in Figure 9 to gauge whether the GZ2D sample is missing any quiescent disks.

The number ratio between edge-on quiescent disks and face-on quiescent disks is largely uniform (at about five oblique disks per edge-on disk). There are, however, hints of a small dearth in the GZ2D sample at low masses. Averaging the number ratios at low masses $\left(\log M_{*}<10.6\right)$ reveals that we find $\approx 10 \%$ fewer GZ2D galaxies compared to the average number ratios of the high-mass quiescent disks. The total number of lowmass quiescent galaxies in our sample is $\approx 900$, so this suggests we may be missing $\approx 90$ low-mass quiescent disk galaxies. 

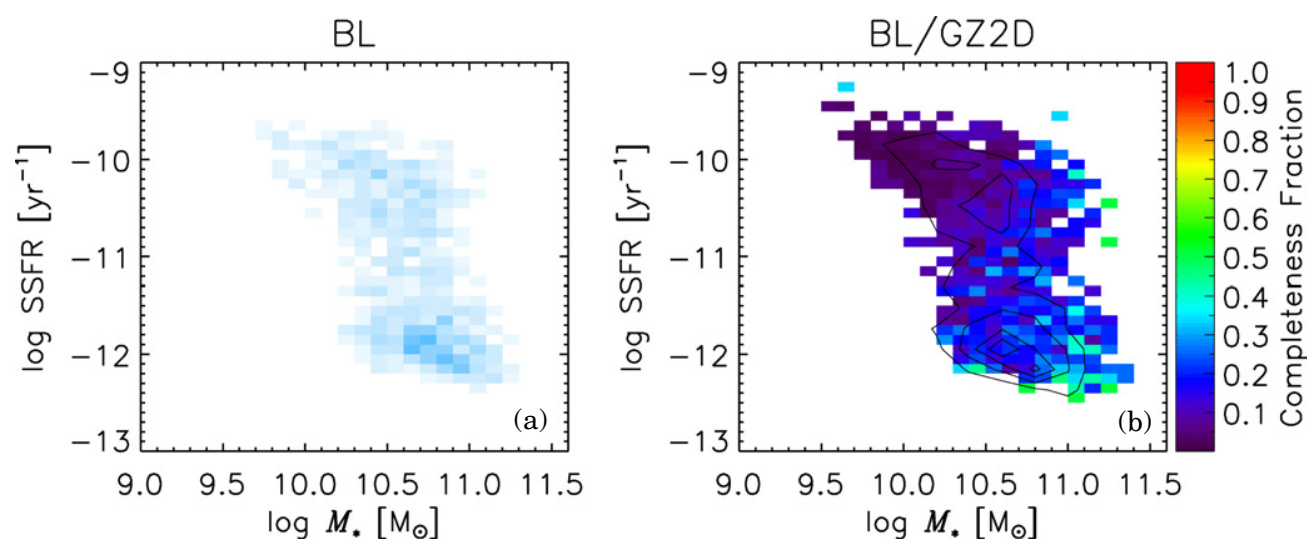

Figure 10. (a) Number density distribution of the BL sample scaled to a quarter of Figure 8(a). (b) Completeness of the BL sample relative to the GZ2D sample; the black contours in this panel represents the number density distribution of the BL sample. Only bins with two or more GZ2D galaxies are shown.

(A color version of this figure is available in the online journal.)
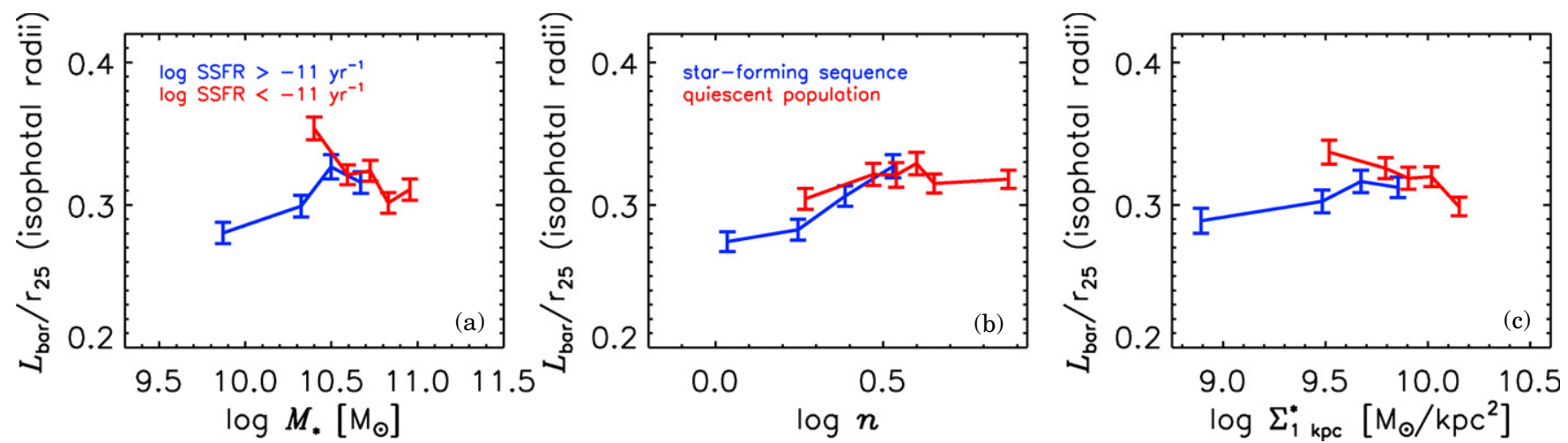

Figure 11. Average bar length scaled by the isophotal radii, $L_{\mathrm{bar}} / \mathrm{r}_{25}$, plotted against (a) $M_{*}$, (b) $n$, and (c) $\Sigma_{1 \mathrm{kpc}}^{*}$. Galaxies were split by their star formation state, namely, $\log$ SSFR $>-11 \mathrm{yr}^{-1}$ (star forming; blue) and $\log$ SSFR $<-11 \mathrm{yr}^{-1}$ (quiescent; red). Each bin contains $\sim 100$ galaxies. The error bars are given by $\sigma / \sqrt{N}$, where $\sigma$ is the standard deviation of $L_{\mathrm{sbar}}$ per bin and $N$ is the total number of galaxies per bin.

(A color version of this figure is available in the online journal.)
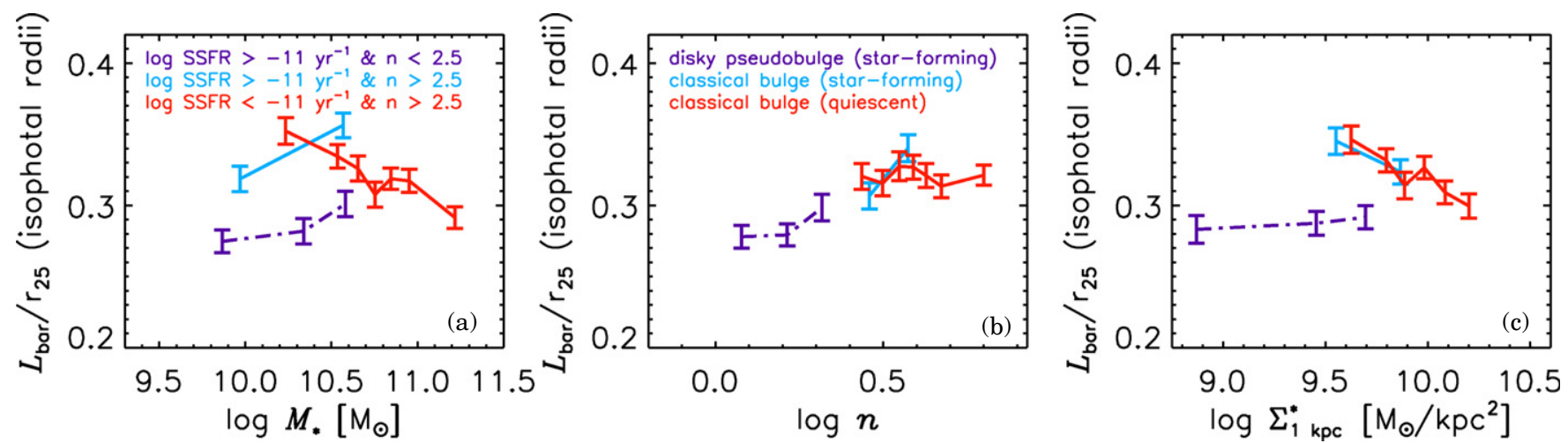

Figure 12. Average $L_{\mathrm{bar}} / \mathrm{r}_{25}$ plotted against (a) $M_{*}$, (b) $n$, and (c) $\Sigma_{1 \mathrm{kpc}}^{*}$. The details are identical to those of Figure 11, with the exception that each bin contains $\sim 75$ galaxies and galaxies are further separated by bulge type, as identified by $n$. Purple points represent the star-forming disky pseudobulge galaxies, light blue points represent the star-forming classical bulge galaxies, and red points represent the quiescent classical bulge galaxies.

(A color version of this figure is available in the online journal.)

Presumably, if a galaxy had a bar, it would be readily spotted and included in this sample; thus, we assume the missing disk galaxies are nonbarred. We assume that the missing disks have values of $n$ and $\Sigma_{1 \mathrm{kpc}}^{*}$ typical for GZ2D galaxies of the same mass and SSFR. We find that the $n$ and $\Sigma_{1 \mathrm{kpc}}^{*}$ values of these low-mass quiescent disks are roughly uniformly distributed, meaning that the $p_{\text {bar }}$ trends with $n$ and $\Sigma_{1 \mathrm{kpc}}^{*}$ for the quiescent population are unaffected by this incompleteness. We can estimate how many unbarred quiescent disk galaxies we are missing for every bin by simply dividing the total number of missing galaxies $(\approx 90)$ by the total number of bins that the quiescent population spans in $n$ and $\Sigma_{1 \mathrm{kpc}}^{*}$, which turns out to be $\sim 20$ bins. Thus, we are missing approximately five unbarred quiescent disks in every $N=100$ bin of $n$ and $\Sigma_{1 \mathrm{kpc}}^{*}$ (see Figures 3(b) and (c)). Even if 


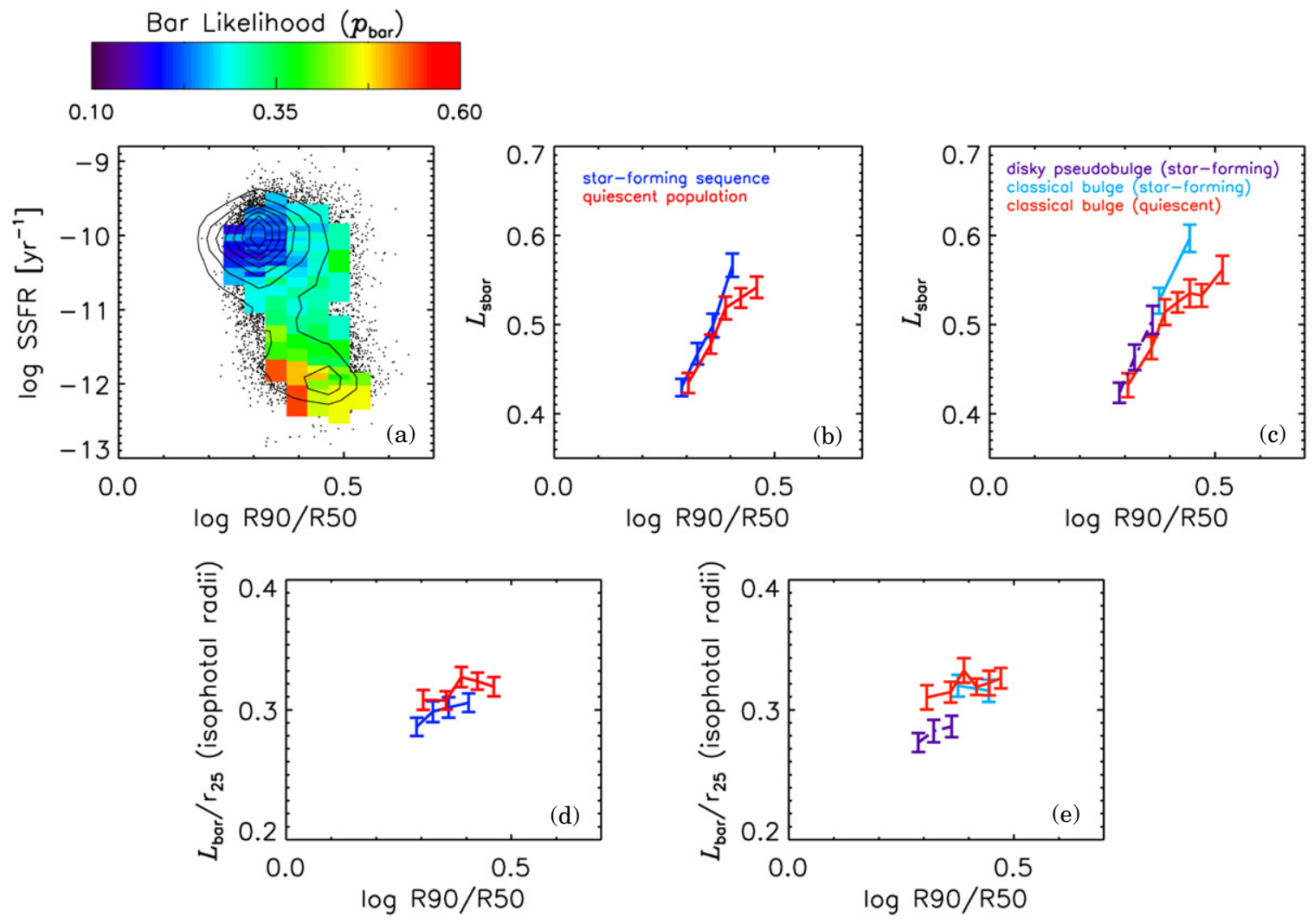

Figure 13. Replacing the Sérsic index with R90/R50. (a) Average $p_{\text {bar }}$ in bins of SSFR and R90/R50. (b) and (c) Bar length scaled by GIM2D bulge+disk model disk scale length versus R90/R50. (d) and (e) Bar length scaled by the isophotal radii versus R90/R50.

(A color version of this figure is available in the online journal.)

all five galaxies have $p_{\text {bar }}=0$, this would reduce the average $p_{\text {bar }}$ in each bin by at most $5 \%$ (by simply adding five more galaxies in the denominator).

The number of missing low-mass disks in the affected part of Figure 3(a)works out to be $\approx 10$ per low-mass quiescent bin (there are $\sim 10$ bins in the low-mass quiescent regime). Figure 3(a) shows that the $p_{\text {bar }}$ values for the low-mass quiescent bins are $\sim 0.60$. Adding 10 nonbarred $\left(p_{\text {bar }}=0\right)$ disk galaxies to these bins, i.e., adding 10 galaxies to the denominator, reduces these $p_{\text {bar }}$ values to $\sim 0.50$. Our qualitative results and interpretation are unaffected. Therefore, the missing nonbarred low-mass quiescent disks do not significantly influence our results.

\section{A.2. Bar Length Sample}

Unlike the GZ2D sample, there is not a concern that the BL sample is missing nonbarred disks since as the sample name implies, the BL sample only contains barred disks. Nevertheless, we want to ensure that it is not suffering any selection bias.

Figure 10 shows the completeness of the BL sample relative to the GZ2D sample. The completeness of BL is approximately bimodal with SSFR. In the high-SSFR regime $(\log$ SSFR > $-11 \mathrm{yr}^{-1}$ ), the BL sample is $\sim 10 \%$ complete, while in the low-SSFR regime $\left(\log\right.$ SSFR $\left.<-11 \mathrm{yr}^{-1}\right)$, it is $\sim 20 \%$.

This completeness bimodality is reasonable because the $\mathrm{BL}$ sample is primarily composed of strong bars, which as illustrated in Figure 3, mainly lie in the quiescent population. However, since our analysis splits the BL sample into star forming and quiescent (i.e., Figures 4 and 7), this difference in completeness should be inconsequential to our results and interpretations.

\section{APPENDIX B}

\section{BAR LENGTH SCALED BY ISOPHOTAL RADII}

Comparing the trends of BL scaled by the isophotal radii (Figures 11 and 12) to those of $L_{\text {sbar }}$ (Figures 4 and 7) shows good agreement. The only noticeable differences are at the highest $n$ and $\Sigma_{1 \mathrm{kpc}}^{*}$, which is hard to interpret and may be due to a number of issues. These GIM2D disk scale lengths may be affected by the prominent bars present in these galaxies. However, the better sky background determination and better object deblending of the GIM2D decompositions could also lead to a more accurate measurement of the disk scale length. More work needs to be done to truly understand the differences, but that is outside the scope of this paper. Moreover, this paper does not put a strong emphasis on the BL trends at the highest $n$ and $\Sigma_{1 \mathrm{kpc}}^{*}$; thus, it does not affect our major conclusions.

\section{APPENDIX C$$
\text { R90/R50 }
$$

Figure 13 shows the effects of using the Petrosian concentration index from SDSS, R90/R50, where R90 and R50 are the radii enclosing $90 \%$ and $50 \%$ of the galaxy luminosity, respectively. The trends with $p_{\text {bar }}$ in Figure 13(a) are almost identical to those with the Sérsic index (Figure 3(b)). Figures 13(b) and (c) show that the trends with R90/R50 for the star-forming sequence, star-forming disky pseudobulge galaxies, and the star-forming classical bulge galaxies are the same as with $n$ (Figures 4(b) and 7(b)), i.e., $L_{\text {sbar }}$ increases with increasing $n$ or R90/R50. 
For the quiescent population (Figure 13(b)) and the quiescent classical bulge galaxies (Figure 13(c)), however, there is a noticeable difference between the trends of $L_{\mathrm{sbar}}$ at the highest values of R90/R50 and $n$. Namely, while there is a decrease of $L_{\mathrm{sbar}}$ at the highest $n$ (Figures 4(b) and 7(b)), there seems to be a steady increase of $L_{\mathrm{sbar}}$ with increasing R90/R50. It is unclear why this is the case. It could be due to the improved sky background determination and object deblending in the fits of Simard et al. (2011) compared to the standard SDSS pipeline. However, whatever the reason, this minor difference does not affect the paper since we leave the interpretation of the BL trends for the highest $n$ values open.

Comparing the trends of BL scaled by the isophotal radii between $n$ (Figures 11(b) and 12(b)) and R90/R50 (Figures 13(d) and (e)) shows general agreement between all populations.

Thus, the results from R90/R50 and $n$ are largely similar, and the use of either would not change the main conclusions of the paper.

\section{REFERENCES}

Abazajian, K. N., Adelman-McCarthy, J. K., Agüeros, M. A., et al. 2009, ApJS, 182,543

Abraham, R. G., Merrifield, M. R., Ellis, R. S., Tanvir, N. R., \& Brinchmann, J. 1999, MNRAS, 308, 569

Aguerri, J. A. L., Méndez-Abreu, J., \& Corsini, E. M. 2009, A\&A, 495, 491

Athanassoula, E. 1992, MNRAS, 259, 345

Athanassoula, E. 2003, MNRAS, 341, 1179 (A03)

Athanassoula, E. 2005, MNRAS, 358, 1477

Athanassoula, E. 2012, in Secular Evolution of Galaxies, ed. J. Falcon-Barroso \& J. H. Knapen (Cambridge: Cambridge Univ. Press), 305

Athanassoula, E., Lambert, J. C., \& Dehnen, W. 2005, MNRAS, 363, 496

Athanassoula, E., Machado, R. E. G., \& Rodionov, S. A. 2013, MNRAS, 429, 1949 (AMR13)

Athanassoula, E., \& Martinet, L. 1980, A\&A, 87, L10

Athanassoula, E., \& Misiriotis, A. 2002, MNRAS, 330, 35

Athanassoula, E., \& Sellwood, J. A. 1986, MNRAS, 221, 213

Bamford, S. P., Nichol, R. C., Baldry, I. K., et al. 2009, MNRAS, 393, 1324

Barazza, F. D., Jogee, S., \& Marinova, I. 2008, ApJ, 675, 1194

Barro, G., Faber, S. M., Pérez-González, P. G., et al. 2013, ApJ, 765, 104

Bell, E. F. 2008, ApJ, 682, 355

Bell, E. F., \& de Jong, R. S. 2001, ApJ, 550, 212

Bell, E. F., McIntosh, D. H., Barden, M., et al. 2004, ApJL, 600, L11

Bell, E. F., van der Wel, A., Papovich, C., et al. 2012, ApJ, 753, 167

Berentzen, I., Heller, C. H., Shlosman, I., \& Fricke, K. J. 1998, MNRAS, 300,49

Berentzen, I., Shlosman, I., Martinez-Valpuesta, I., \& Heller, C. H. 2007, ApJ, 666,189

Blanton, M. R., Eisenstein, D., Hogg, D. W., Schlegel, D. J., \& Brinkmann, J. 2005a, ApJ, 629, 143

Blanton, M. R., Hogg, D. W., Bahcall, N. A., et al. 2003, ApJ, 594, 186

Blanton, M. R., Schlegel, D. J., Strauss, M. A., et al. 2005b, AJ, 129, 2562

Block, D. L., Buta, R., Knapen, J. H., et al. 2004, AJ, 128, 183

Brinchmann, J., Charlot, S., White, S. D. M., et al. 2004, MNRAS, 351, 1151

Cameron, E., Carollo, C. M., Oesch, P., et al. 2010, MNRAS, 409, 346

Catinella, B., Schiminovich, D., Kauffmann, G., et al. 2010, MNRAS, 403, 683

Cheung, E., Faber, S. M., Koo, D. C., et al. 2012, ApJ, 760, 131

Coelho, P., \& Gadotti, D. A. 2011, ApJL, 743, L13

Combes, F., \& Elmegreen, B. G. 1993, A\&A, 271, 391

Courteau, S., de Jong, R. S., \& Broeils, A. H. 1996, ApJL, 457, L73

Darg, D. W., Kaviraj, S., Lintott, C. J., et al. 2010, MNRAS, 401, 1043

Davis, M., Guhathakurta, P., Konidaris, N. P., et al. 2007, ApJL, 660, L1

de Souza, R. E., Gadotti, D. A., \& dos Anjos, S. 2004, ApJS, 153, 411

Debattista, V. P., Mayer, L., Carollo, C. M., et al. 2006, ApJ, 645, 209

Debattista, V. P., \& Sellwood, J. A. 2000, ApJ, 543, 704

Dressel, L. L. 1988, ApJL, 329, L69

Drory, N., \& Fisher, D. B. 2007, ApJ, 664, 640

Ellison, S. L., Nair, P., Patton, D. R., et al. 2011, MNRAS, 416, 2182

Elmegreen, B. G., Bournaud, F., \& Elmegreen, D. M. 2008, ApJ, 688, 67

Elmegreen, B. G., \& Elmegreen, D. M. 1985, ApJ, 288, 438

Elmegreen, B. G., Elmegreen, D. M., \& Hirst, A. C. 2004, ApJ, 612, 191

Elmegreen, B. G., Elmegreen, D. M., Knapen, J. H., et al. 2007, ApJL, 670, L97

Erwin, P. 2005, MNRAS, 364, 283
Eskridge, P. B., Frogel, J. A., Pogge, R. W., et al. 2000, AJ, 119, 536

Fang, J. J., Faber, S. M., Koo, D. C., \& Dekel, A. 2013, ApJ, 776, 63

Fisher, D. B., \& Drory, N. 2008, AJ, 136, 773

Fisher, D. B., \& Drory, N. 2010, ApJ, 716, 942

Franx, M., van Dokkum, P. G., Schreiber, N. M. F., et al. 2008, ApJ, 688, 770

Freeman, K. C. 1970, ApJ, 160, 811

Friedli, D., \& Benz, W. 1993, A\&A, 268, 65

Friedli, D., \& Benz, W. 1995, A\&A, 301, 649

Friedli, D., Benz, W., \& Kennicutt, R. 1994, ApJL, 430, L105

Gadotti, D. A. 2009, MNRAS, 393, 1531

Gadotti, D. A. 2011, MNRAS, 415, 3308

Giuricin, G., Tamburini, L., Mardirossian, F., Mezzetti, M., \& Monaco, P. 1994, ApJ, 427, 202

Graham, A. W., \& Driver, S. P. 2005, PASA, 22, 118

Hao, L., Jogee, S., Barazza, F. D., Marinova, I., \& Shen, J. 2009, in ASP Conf. 419, Galaxy Evolution: Emerging Insights and Future Challenges, ed. S. Jogee, I. Marinova, L. Hao, \& G. A. Blanc (San Francisco, CA: ASP), 402

Hawarden, T. G., Mountain, C. M., Leggett, S. K., \& Puxley, P. J. 1986, MNRAS, $221,41 \mathrm{P}$

Heller, C. H., \& Shlosman, I. 1994, ApJ, 424, 84

Herrington, J., Bell, E. F., McIntosh, D. H., et al. 2013, ApJ, submitted

Ho, L. C., Filippenko, A. V., \& Sargent, W. L. W. 1997, ApJ, 487, 591

Hohl, F. 1971, ApJ, 168, 343

Holley-Bockelmann, K., Weinberg, M., \& Katz, N. 2005, MNRAS, 363, 991

Hoyle, B., Masters, K. L., Nichol, R. C., et al. 2011, MNRAS, 415, 3627

Huang, J. H., Gu, Q. S., Su, H. J., et al. 1996, A\&A, 313, 13

Hubble, E. P. 1936, Realm of the Nebulae, ed. E. P. Hubble (New Haven, CT: Yale Univ. Press)

Inoue, S., \& Saitoh, T. R. 2012, MNRAS, 422, 1902

Jogee, S. 2006, in Physics of Active Galactic Nuclei at all Scales, ed. D. Alloin, R. Johnson, \& P. Lira (Lecture Notes in Physics, Vol. 693; Berlin: Springer), 143

Jogee, S., Barazza, F. D., Rix, H.-W., et al. 2004, ApJL, 615, L105

Kauffmann, G., Heckman, T. M., White, S. D. M., et al. 2003, MNRAS, 341,33

Kauffmann, G., Li, C., Fu, J., et al. 2012, MNRAS, 422, 997

Knapen, J. H., Beckman, J. E., Heller, C. H., Shlosman, I., \& de Jong, R. S. 1995, ApJ, 454, 623

Knapen, J. H., Shlosman, I., \& Peletier, R. F. 2000, ApJ, 529, 93

Kormendy, J. 1979, ApJ, 227, 714

Kormendy, J. 1993, in IAU Symp. 153, Galactic Bulges, ed. H. DeJonghe \& H. J. Habing (Dordrecht: Kluwer), 209

Kormendy, E. 2012, in Secular Evolution of Galaxies, ed. J. Falcon-Barroso \&

J. H. Knapen (Cambridge: Cambridge Univ. Press), 1

Kormendy, J., \& Kennicutt, R. C. 2004, ARA\&A, 42, 603

Kraljic, K., Bournaud, F., \& Martig, M. 2012, ApJ, 757, 60

Kroupa, P. 2001, MNRAS, 322, 231

Laine, S., Shlosman, I., Knapen, J. H., \& Peletier, R. F. 2002, ApJ, 567, 97

Land, K., Slosar, A., Lintott, C., et al. 2008, MNRAS, 388, 1686

Laurikainen, E., Salo, H., \& Buta, R. 2004, ApJ, 607, 103

Laurikainen, E., Salo, H., \& Buta, R. 2005, MNRAS, 362, 1319

Laurikainen, E., Salo, H., Buta, R., \& Knapen, J. H. 2007, MNRAS, 381, 401

Laurikainen, E., Salo, H., Buta, R., \& Knapen, J. H. 2009, ApJL, 692, L34

Laurikainen, E., Salo, H., \& Rautiainen, P. 2002, MNRAS, 331, 880

Lee, G.-H., Park, C., Lee, M. G., \& Choi, Y.-Y. 2012, ApJ, 745, 125

Lintott, C., Schawinski, K., Bamford, S., et al. 2011, MNRAS, 410, 166

Lintott, C. J., Schawinski, K., Slosar, A., et al. 2008, MNRAS, 389, 1179

Lynden-Bell, D., \& Kalnajs, A. J. 1972, MNRAS, 157, 1

MacArthur, L. A., Courteau, S., \& Holtzman, J. A. 2003, ApJ, 582, 689

Martel, H., Kawata, D., \& Ellison, S. L. 2013, MNRAS, 431, 2560

Martin, P. 1995, AJ, 109, 2428

Martin, P., \& Friedli, D. 1997, A\&A, 326, 449

Martin, P., \& Roy, J.-R. 1994, ApJ, 424, 599

Martinet, L., \& Friedli, D. 1997, A\&A, 323, 363

Martinez-Valpuesta, I., Shlosman, I., \& Heller, C. 2006, ApJ, 637, 214

Martini, P., Regan, M. W., Mulchaey, J. S., \& Pogge, R. W. 2003, ApJ, 589, 774

Masters, K. L., Nichol, R. C., Hoyle, B., et al. 2011, MNRAS, 411, 2026

Masters, K. L., Nichol, R. C., Haynes, M. P., et al. 2012, MNRAS, 424, 2180

Matsuda, T., \& Nelson, A. H. 1977, Natur, 266, 607

Melvin, T., Masters, K., Lintott, C., et al. 2013, MNRAS, submitted

Menéndez-Delmestre, K., Sheth, K., Schinnerer, E., Jarrett, T. H., \& Scoville, N. Z. 2007, ApJ, 657, 790

Minchev, I., Famaey, B., Combes, F., et al. 2011, A\&A, 527, A147

Mulchaey, J. S., \& Regan, M. W. 1997, ApJL, 482, L135

Nair, P. B., \& Abraham, R. G. 2010a, ApJS, 186, 427

Nair, P. B., \& Abraham, R. G. 2010b, ApJL, 714, L260

Noguchi, M. 1988, A\&A, 203, 259 
Oh, S., Oh, K., \& Yi, S. K. 2012, ApJS, 198, 4

Okamoto, T. 2013, MNRAS, 428, 718

O’Neill, J. K., \& Dubinski, J. 2003, MNRAS, 346, 251

Peng, C. Y., Ho, L. C., Impey, C. D., \& Rix, H.-W. 2002, AJ, 124, 266

Regan, M. W., \& Elmegreen, D. M. 1997, AJ, 114, 965

Saintonge, A., Kauffmann, G., Kramer, C., et al. 2011, MNRAS, 415, 32

Sakamoto, K., Okumura, S. K., Ishizuki, S., \& Scoville, N. Z. 1999, ApJ, 525,691

Salim, S., Rich, R. M., Charlot, S., et al. 2007, ApJS, 173, 267

Sánchez-Janssen, R., \& Gadotti, D. A. 2013, MNRAS, 432, 56

Sanders, R. H., \& Huntley, J. M. 1976, ApJ, 209, 53

Schawinski, K., Urry, M. C., Simmons, B. D., et al. 2013, MNRAS, submitted

Schiminovich, D., Wyder, T. K., Martin, D. C., et al. 2007, ApJS, 173, 315

Schwarz, M. P. 1981, ApJ, 247, 77

Scoville, N., Aussel, H., Brusa, M., et al. 2007, ApJS, 172, 1

Sellwood, J. A. 1980, A\&A, 89, 296

Sellwood, J. A. 2013, arXiv:1310.0403

Sellwood, J. A., \& Wilkinson, A. 1993, RPPh, 56, 173

Shen, J., \& Sellwood, J. A. 2004, ApJ, 604, 614

Shen, S., Mo, H. J., White, S. D. M., et al. 2003, MNRAS, 343, 978

Sheth, K., Elmegreen, D. M., Elmegreen, B. G., et al. 2008, ApJ, 675, 1141

Sheth, K., Melbourne, J., Elmegreen, D. M., et al. 2012, ApJ, 758, 136

Sheth, K., Vogel, S. N., Regan, M. W., Thornley, M. D., \& Teuben, P. J. 2005, ApJ, 632, 217
Shlosman, I., Frank, J., \& Begelman, M. C. 1989, Natur, 338, 45

Shlosman, I., \& Noguchi, M. 1993, ApJ, 414, 474

Simard, L., Mendel, J. T., Patton, D. R., Ellison, S. L., \& McConnachie, A. W. 2011, ApJS, 196, 11

Simard, L., Willmer, C. N. A., Vogt, N. P., et al. 2002, ApJS, 142, 1

Simkin, S. M., Su, H. J., \& Schwarz, M. P. 1980, ApJ, 237, 404

Simmons, B. D., Lintott, C., Schawinski, K., et al. 2013, MNRAS, 429, 2199

Skibba, R. A., Bamford, S. P., Nichol, R. C., et al. 2009, MNRAS, 399, 966

Skibba, R. A., Masters, K. L., Nichol, R. C., et al. 2012, MNRAS, 423, 1485

Strauss, M. A., Weinberg, D. H., Lupton, R. H., et al. 2002, AJ, 124, 1810

Tremaine, S., \& Weinberg, M. D. 1984, MNRAS, 209, 729

Valenzuela, O., \& Klypin, A. 2003, MNRAS, 345, 406

Vila-Costas, M. B., \& Edmunds, M. G. 1992, MNRAS, 259, 121

Villa-Vargas, J., Shlosman, I., \& Heller, C. 2010, ApJ, 719, 1470

Wada, K., \& Habe, A. 1992, MNRAS, 258, 82

Wada, K., \& Habe, A. 1995, MNRAS, 277, 433

Wake, D. A., van Dokkum, P. G., \& Franx, M. 2012, ApJL, 751, L44

Wang, J., Kauffmann, G., Overzier, R., et al. 2012, MNRAS, 423, 3486

Willett, K. W., et al. 2013, MNRAS, submitted

Williams, C. C., Giavalisco, M., Cassata, P., et al. 2013, arXiv:1310:3819

Williams, M. J., Bureau, M., \& Kuntschner, H. 2012, MNRAS, 427, L99

Wuyts, S., Förster Schreiber, N. M., van der Wel, A., et al. 2011, ApJ, 742, 96

Zaritsky, D., Kennicutt, R. C., Jr., \& Huchra, J. P. 1994, ApJ, 420, 87 